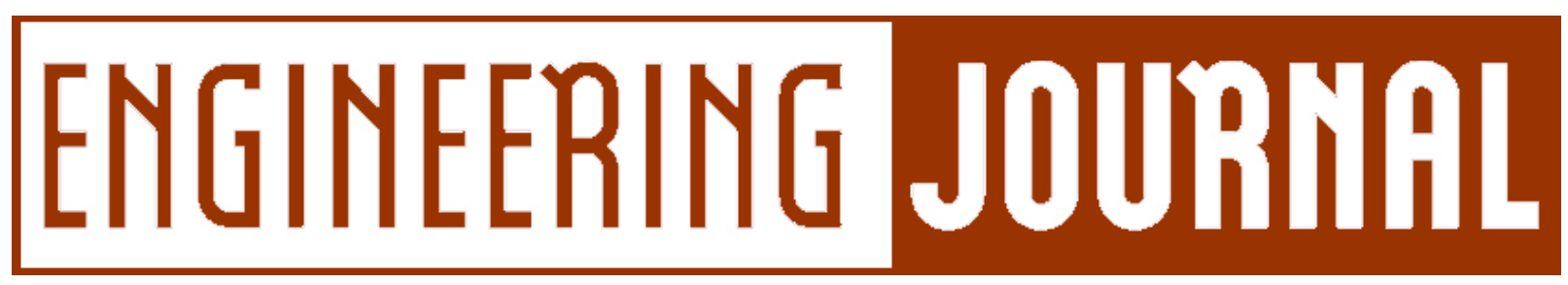

Review

\title{
Evolution Toward 5G Mobile Networks - A Survey on Enabling Technologies
}

\author{
Rony Kumer Saha ${ }^{1, \mathrm{a}}$, Poompat Saengudomlert ${ }^{2, \mathrm{~b}}$, Chaodit Aswakul ${ }^{1, \mathrm{c}, *}$ \\ 1 Department of Electrical Engineering, Faculty of Engineering, Chulalongkorn University, Bangkok 10330, \\ Thailand \\ 2 Bangkok University-Center of Research in Optoelectronics, Communications, and Control Systems (BU- \\ CROCCS), Bangkok University, Thailand \\ E-mail: arony107976@gmail.com, bpoompat.s@bu.ac.th, cchaodit.a@chula.ac.th (Corresponding author)
}

\begin{abstract}
In this paper, an extensive review has been carried out on the trends of existing as well as proposed potential enabling technologies that are expected to shape the fifth generation $(5 G)$ mobile wireless networks. Based on the classification of the trends, we develop a $5 \mathrm{G}$ network architectural evolution framework that comprises three evolutionary directions, namely, (1) radio access network node and performance enabler, (2) network control programming platform, and (3) backhaul network platform and synchronization. In (1), we discuss node classification including low power nodes in emerging machine-type communications, and network capacity enablers, e.g., millimeter wave communications and massive multiple-input multiple-output. In (2), both logically distributed cell/device-centric platforms, and logically centralized conventional/wireless software defined networking control programming approaches are discussed. In (3), backhaul networks and network synchronization are discussed. A comparative analysis for each direction as well as future evolutionary directions and challenges toward 5G networks are discussed. This survey will be helpful for further research exploitations and network operators for a smooth evolution of their existing networks toward 5G networks.
\end{abstract}

Keywords: 5G mobile network, survey, network node, network control, backhaul network, synchronization.

ENGINEERING JOURNAL Volume 20 Issue 1

Received 20 March 2015

Accepted 26 June 2015

Published 29 January 2016

Online at http://www.engj.org/

DOI:10.4186/ej.2016.20.1.87 


\section{Introduction}

\subsection{Incentives and Requirements of 5G Networks}

The exponentially growing number of mobile users and an ever increasing demand of high data rates impose several challenges on current cellular networks such as the fourth generation (4G) long term evolution (LTE) network and its evolutions in terms of a high network capacity and a wide coverage area to meet up user demands in future 5G networks. The major drawbacks that mobile users face from current networks are a nonuniform low data rate, a non-unified quality of experience (QoE), a poor end-to-end performance, a weak indoor coverage, an insufficient high mobility performance, and a high cost per bit transfer. Similarly, network operators face a number of difficulties in providing satisfactory services from current networks, e.g., provisions of a high network capacity, a low latency, a high spectral efficiency, a large spectrum availability, and a low energy consumption.

Internet data traffic through mobile wireless access networks is expected to grow faster than that through traditional fixed access networks [1]. According to [2], the growth of data usages will continue, and an incremental approach to serve user demands will not be sufficient toward meeting mobile network demands by 2020 [3]. Internet protocol (IP) data usage by wireless networks will increase from under 3 exabytes in 2010 to over 500 exabytes by 2020 [3]. Internet of Things (IoT) is expected to dominate over the current practice of human-centric Internet in $5 \mathrm{G}$ networks [4]. Nonetheless, with existing networks, it is not possible to address these aforementioned challenges. In this context, 5G mobile network technologies are expected to be standardized around 2020. To address 5G requirements and technologies, there has already been a great attention in research communities. Though there is no exact specification on what $5 \mathrm{G}$ networks will encompass, based on what most people have agreed so far as compared to $4 \mathrm{G}$ networks, $5 \mathrm{G}$ networks should attain the followings as given in Table $1[3,5-6]$.

Table 1. 5G network requirements.

\begin{tabular}{ll}
\hline \multicolumn{1}{c}{ Attributes } & \multicolumn{1}{c}{ Values (compared to 4G networks) } \\
\hline System capacity & 1000 times \\
Spectral efficiency & 10 times \\
Energy efficiency & 10 times \\
Longer battery life time & 10 times \\
Reduced latency & 5 times \\
Higher number of connecting devices & $10-100$ times \\
Reduction in energy per bit as well as cost per bit & 100 times \\
Mobility support & up to $500 \mathrm{~km} / \mathrm{hr}$ \\
\hline
\end{tabular}

\subsection{State-of-the-Art Practice, Challenge and Evolutionary Direction}

Network performances play a significant role on the evolution of a new generation of mobile networks. In mobile wireless networks, the network capacity has been seen as the main driver for evolutions of a number of past generations, e.g., second generation $(2 \mathrm{G})$, third generation $(3 \mathrm{G})$, and $4 \mathrm{G}$. In a similar trend, achieving a high capacity is also the major driver for the evolution of $5 \mathrm{G}$ networks. Typically, to address a high capacity demand, network operators consider reducing the distance between a base station (BS) and a user equipment (UE) in order to improve the received signal strength at a UE. A standard practice to reduce cell coverage is to deploy low power nodes such as femtocells, picocells in the coverage of a macrocell. This results in shifting traditional homogeneous networks (HomNets) to more advanced heterogeneous networks (HetNets). A low power node covers a small portion of a macrocell area, and hence is also termed as a small cell.

Since typically the capacity per unit area increases with the number of small cells, small cells are expected to be deployed as densely as possible, and hence HetNets are thus extended to dense HetNets as one of the potential techniques to address the high capacity demand of $5 \mathrm{G}$ networks. Besides dense HetNets, the capacity of $5 \mathrm{G}$ networks will most likely be driven by spectrum aggregation and spectral efficiency techniques. Since 
microwave frequencies deployed in existing networks are almost saturated, millimeter wave (mmWave) spectrum bands are considered for $5 \mathrm{G}$ networks to increase the system bandwidth because of usable high spectrum availability in these bands. Many advanced techniques such as enhanced inter-cell interference coordination (eICIC) [7], cooperative communication, networked multiple-input multiple-output (MIMO), and massive MIMO have been proposed as the major enabling technologies to improve the spectral efficiency of $5 \mathrm{G}$ networks. In addition, for the proper coordination among nodes to enable these advanced technologies, particularly the cooperative communication in HetNets, an appropriate backhaul network and network synchronization must be provided. There have been active researches on these aforementioned advanced technologies to meet the high capacity demand of $5 \mathrm{G}$ networks.

One of the noticeable characteristics of current mobile networks is that both control decision and processing task units exist in the same networking device, e.g., BS, serving gateway (SGW), and packet data network gateway (PGW) nodes in an LTE network. This implies that current mobile networks are based on the distributed and coupled control and data planes where each entity distributed over the network is responsible for executing both decision making and processing tasks. This distributed nature of current mobile networks results in manifold challenges as follows:

1) complexity in control and management of a network (e.g., a mobility manager needs to coordinate more with an increase in the number of users)

2) a poor network efficiency (e.g., it is difficult to update existing devices with advanced solutions)

3) non-evolvability (e.g., there are fewer scopes in creating service differentiations between competitors)

4) inflexibility (e.g., operators rely on vendor specific solutions to introduce a new feature)

Hence, to address these challenges, network operators seek to new solutions [1], and rather than considering the traditional cell-centric control, an advanced device-centric control has been proposed as a solution [8] in distributed networks. The other approach is to apply software defined networking (SDN) concept as a new solution to wireless networks in order to address the aforementioned issues. For example, since elements of existing networks hold both control and data plane functionalities, these two planes can be separated by introducing SDN. SDN then shifts network-wide control functionalities to a logically centralized but physically distributed entity called SDN controller such that distributed control complexities of existing networks can be overcome. The logically centralized controlled architecture provides network programmable capabilities centrally by the controller. However, in a distributed controlled approach, a network is programmed at each networking device distributed network wide, e.g., BSs in radio access networks and SGW and PGW in core networks of LTE systems. In a logically centralized network programming approach, by having the global view of a network, a controller can manage various functionalities such as radio resource management, interference mitigation, load balancing, and mobility control among network nodes more efficiently than the distributed ones. Hence, the network programmable aspect has a remarkable impact on the overall network control and management performances and is seen as an eventual path toward the evolution of $5 \mathrm{G}$ networks.

\subsection{G Network Architectural Evolution Framework}

The $5 \mathrm{G}$ cellular is in its early stage of research and development with no clear direction on how the envisaged high capacity of $5 G$ networks can be achieved. Though several proposals on enabling technologies as aforementioned from various aspects have been introduced in literature, how all these technologies can be applied, controlled, and managed to meet the required $5 \mathrm{G}$ capacity as well as what the $5 \mathrm{G}$ network architecture will eventually look like to achieve the target capacity is still an open research issue. Based on the above trends of enabling technologies, $5 \mathrm{G}$ networks are expected to evolve in three major directions, namely, radio access network nodes and performance improvement technologies to address the high $5 \mathrm{G}$ capacity demand, network programmable capability for the network control, and backhaul networks and network synchronization to enable coordination between nodes for a cost-effective QoE performance. Hence, we classify all these trends into three such directions as radio access network node and performance enabler, network control programming platform, and backhaul network platform and synchronization as parallel horizontal evolutionary paths toward 5G networks. All these evolutionary paths are to be vertically integrated to incorporate all evolutionary aspects for a full-fledge $5 \mathrm{G}$ network architectural evolution. 
A few studies have been done towards this direction, such as [9-10]. However, most of the existing survey articles consider mainly one of the three evolution directions with some additional insights on the others. For example, [9] mainly addressed emerging technologies of the radio access network node and performance enabler with an additional insight on SDN. Different from [9], [10] addressed a number of radio access network nodes and performance enablers, network virtualization along with additional insights on research challenges regarding such issues as $5 \mathrm{G}$ measurement and testing and radio resource management. Although the issues regarding $5 \mathrm{G}$ backhaul networks are significant to enable emerging technologies of other evolutionary directions, these issues remain mostly unaddressed in the existing literatures such as [9-10]. In addition, there is a lack of an architectural evolution framework for $5 \mathrm{G}$ networks that can capture most of the dominant enabling technologies in a systematic approach. In this paper, we aim to address these issues through a systematic framework that captures most of the dominant enabling technologies of the $5 \mathrm{G}$ network architectural evolution from a broad set of perspectives.

A framework of the $5 \mathrm{G}$ network architectural evolution for the network capacity is proposed in Fig. 1 and is detailed in Fig. 2. An extensive review on enabling technologies in all these directions from various perspectives is carried out to give an insight on how all these directions will fit in together under different requirements and constraints, and what would be the possible best match of these directions in terms of enabling technologies to meet the $5 \mathrm{G}$ capacity demand. The survey will allow network operators to take the best choice on enabling technique and technology alternatives to meet the high capacity demand of $5 \mathrm{G}$ networks.

The paper is organized as follows. In Section 2, we discuss radio access network nodes and performance enablers in terms of low power nodes and enabling technologies for the capacity and performance improvement. Network control programing platforms in both distributed and centralized approaches are discussed in Section 3. Backhaul network platforms and network synchronizations are discussed in Section 4, followed by a discussion on future evolutionary directions and challenges toward $5 \mathrm{G}$ networks in Section 5. We draw an overall conclusion in Section 6.

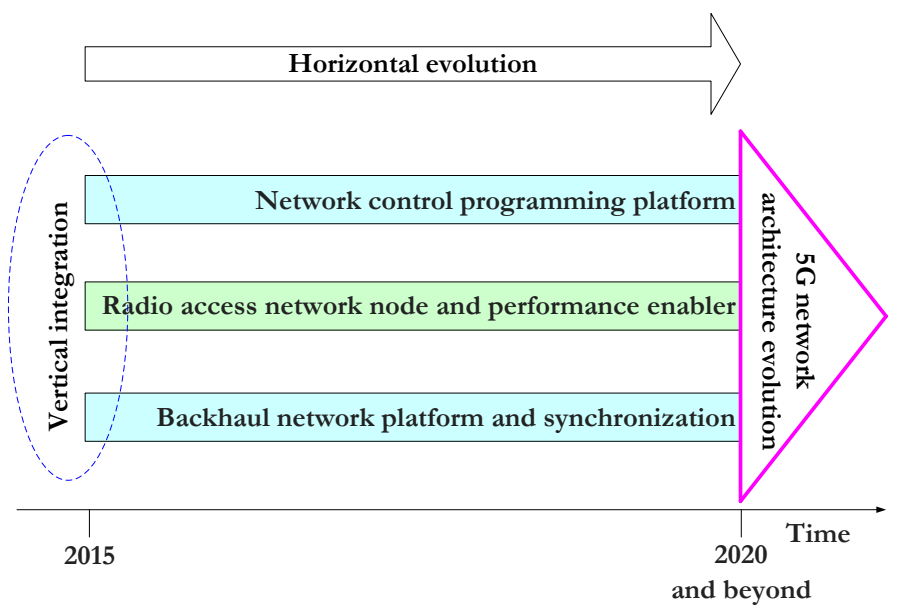

Fig. 1. $5 \mathrm{G}$ network architectural evolution framework. 


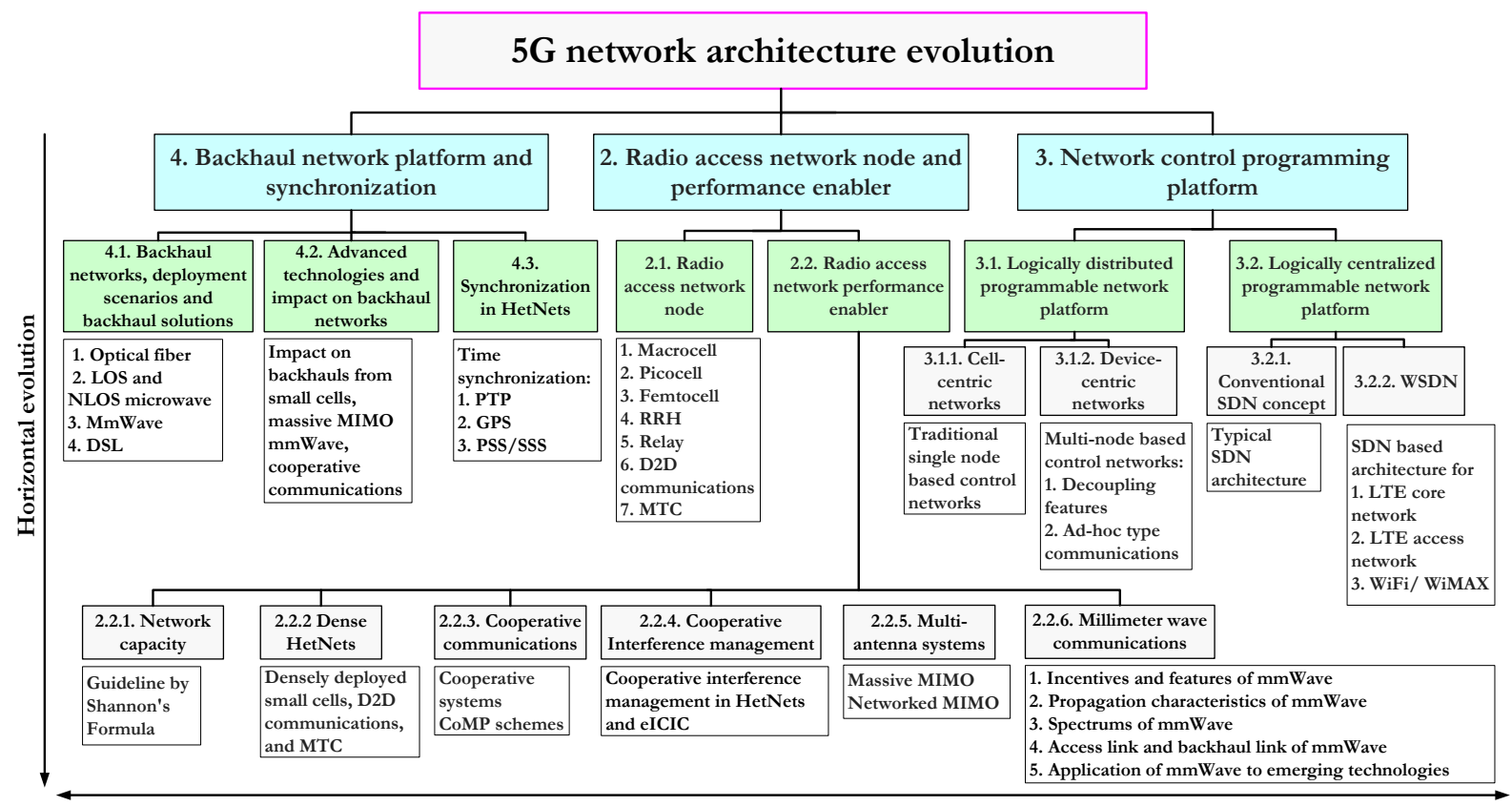

Vertical integration

Fig. 2. Detailed 5G network architectural evolution framework.

\section{Radio Access Network Node and Performance Enabler}

\subsection{Radio Access Network Node}

The $5 \mathrm{G}$ radio access network is expected to consist of densely deployed heterogeneous nodes, particularly low power nodes. Network densification with small cells is considered as one of the prominent approaches to achieve the high capacity of $5 \mathrm{G}$ networks where small cells are deployed in the coverage of a macrocell such that the distance dependent path loss between a UE and a small cell BS is reduced to improve the throughput per user. Various types of small cells such as picocell, femtocell, relay node, and remote radio head (RRH) have been standardized by the 3GPP for 4G LTE-Advanced systems with a future deployment of device-to-device (D2D) and machine type communications as parts of beyond LTE-Advanced or 5G systems. Each of these small cells has a number of different characteristics from others in terms of transmission power, cell coverage area, and so on. Hence, 5G network architectures depend largely on the type and the density of these small cells. In the following, the basic features of each of these small cells and the macrocell are discussed.

A macrocell is usually characterized by a high transmission power, a wide cell coverage, a large number of concurrent user associations, and an open access for all users. Antenna radiation patterns of macro BSs can be either omnidirectional or sectorized. The number of antennas at a macro BS can be multiple, e.g., an evolved node BS (eNB) of LTE-Advanced systems with the maximum number of eight antennas in the downlink. These eNBs are connected to each other via X2 backhauls to exchange signaling information, e.g., common control signals using the C-plane and user data information using the U-plane for cooperation.

A picocell is a BS with a relatively lower transmission power than that of an $\mathrm{eNB}$, and correspondingly with a limited cell coverage. A picocell usually serves a few tens of users, and is deployed typically in hotspot areas such as shopping mall, bus station. Picocells are usually connected to an eNB with X2 backhauls. The major purpose of deploying a picocell is to improve local user throughputs within its periphery and also places where an indoor coverage from a macro BS is poor. Like a macro BS, a pico BS is also deployed by an operator [7].

A femtocell is also a low power BS with a transmission power typically less than that of a picocell. It is also called as home eNB (HeNB) [11] in the 3GPP LTE-Advanced, and usually covers a limited area less than that of a picocell. Based on the access policy, femtocells are usually classified into three types, namely, an open access, a closed subscriber group (CSG), and a hybrid access [12]. In an open access type, any user can get 
access to a femtocell; whereas in a CSG, only a specific group of users are allowed to get an access; and in a hybrid access, a portion of femtocell resources is restricted for a particular group of users, and the remaining can be accessed by any user. Femtocells are usually deployed in indoor coverage areas to improve indoor user throughputs as well as in areas where the received signal strength from a macro BS at a UE is very low or lower than the minimum requirement for establishing and continuing a communication link. Femtocells use consumers' broadband connections as backhaul links such as digital subscriber line (DSL), copper cable, and optical fiber [7].

Similar to a macro BS, a remote radio head (RRH) is a high transmission power BS which is usually compact in size and low weight [7]. RRHs are connected to a macro BS via high speed links such as optical fibers, and all control and baseband signal processing tasks are performed for RRHs at macro BSs. The main purpose of RRHs is to distribute the cell coverage by remote BSs such that constraints from site acquisitions [7] can be flexibly tackled. This distributed form of antennas is called a distributed antenna system (DAS) which offers an improved link quality, reliability, and coverage because of the more frequent presence of line-of-sight (LOS) links.

The relays are also low power nodes that relay signals from a macro BS to UEs and vice versa [7]. Relays are of two categories, namely, fixed relays and mobile relays. For fixed relaying, a relay node uses the wireless backhaul to connect to a macro BS. The link that exists between a UE and a relay node is called an access link, and the link that exists between a macro BS and a relay node is called a backhaul link. Relays can be classified based on a number of aspects, namely, spectrum usage, UE awareness, type, and degree of processing [7, 1314]. For example, based on the usage spectrum in access and backhaul links, relays are classified as inband relays and outband relays. When the relay operation is performed at the same frequency on the access link as that on the backhaul link, the relay is termed as inband relay; however, if performed at a different frequency, the relay is termed as outband relay. For mobile relaying, relays are usually of two types: moving network-relays and mobile user relays [15]. In moving network-relays, dedicated relay nodes are set up on moving vehicles such as trains and buses to exchange data between macro BSs and UEs onboard in order to improve cell coverage in moving vehicles. In mobile user relays, distributed UEs can relay information in an ad-hoc fashion that can complement existing cellular networks. Given a sufficient level of infrastructures, theoretical studies [15] have proved that mobile user relays can improve the sum throughput of users. However, mobile user relays can be disadvantageous from such constraints as power consumption of UE batteries and complicated billing problems.

D2D communications are expected to be deployed in 5G networks [16]. D2D communications have been paid attention by research communities for years [17] and studied in 3GPP Releases 12 and 13 particularly on D2D discovery and communication [18]. Two scenarios are defined in 3GPP [19], namely, direct data path and locally routed data path. In the former scenario, devices exchange information without any involvement of network elements for the U-plane; however in the later scenario, devices involve controlling nodes for relaying data to exchange locally without routing through the core networks. Hence, rather than only macro BSs, other nodes can have control on signal transmissions and receptions with an assistance from the network. Specifically, nodes in cell-edge areas where the communication link between a UE and a macro BS is typically weak but the link between two cell-edge UEs can be very good such that one of these two UEs can act as a transmitter for the other. A macro BS carries out all control signaling operations, e.g., synchronization.

Machine-type communications (MTC) or machine-to-machine (M2M) communications are one of the enabling technologies for $5 \mathrm{G}$ networks that will allow devices such as cars and health monitoring devices to connect to the Internet [20]. Consumer electronic devices can be networked, interconnected, and accessible or controllable remotely. That results in innovation shifting from products to services delivered by these devices [21]. In MTC, a number of devices communicate to one another or to a central controller even without any human intervention [22]. MTC is generally characterized by a small packet size, a low mobility, a group based communication, a secure connection, and a transmission delay tolerant [23-24]. A few applications of MTC are health care, tracking, sensor monitoring, vehicular telematics, and smart grid [25]. For supporting MTC in 4G LTE-Advanced systems, service requirements for network improvements for MTC are specified in [26] by the $3 \mathrm{GPP}$ to address the requirements of $5 \mathrm{G}$ networks [27]. Further, the European Telecommunications Standards Institute (ETSI) has already defined the overall end-to-end M2M functional architecture [28]. 
MTC applications are typically hosted by an application server which may be connected directly with a cellular network or may use service capability servers to offer additional control and data services [29]. Devices in $\mathrm{M} 2 \mathrm{M}$ communications can share and operate on unlicensed spectrums of cellular networks and help reduce transmission loads and improve performances of cellular networks [30-32]. There are a number of scenarios for the convergence of M2M networks and cellular networks as presented in [32]. For example, the most advanced convergence scenario is a fully connected M2M network that allows a single data flow to be routed through multiple M2M connections and all M2M devices can act at a time as cellular consumers as well as M2M communication providers. Table $2[7,14,33-37]$ shows a comparative framework of these nodes.

Table 2. A comparative framework of various node types in HetNets.

\begin{tabular}{|c|c|c|c|c|c|}
\hline \multirow[b]{2}{*}{$\begin{array}{c}\text { Dense } \\
\text { HetNet } \\
\text { nodes }\end{array}$} & \multicolumn{5}{|c|}{ Specifications attributes } \\
\hline & $\begin{array}{c}\text { Typical } \\
\text { transmission } \\
\text { power } \\
\end{array}$ & $\begin{array}{c}\text { Cell } \\
\text { coverage }\end{array}$ & $\begin{array}{l}\text { RAN backhaul } \\
\text { connection }\end{array}$ & Utilization & $\begin{array}{c}\text { Deployment } \\
\text { environment and } \\
\text { planning } \\
\end{array}$ \\
\hline Macrocell & $46-49 \mathrm{dBm}$ & Few kms & $\begin{array}{l}\text { LOS microwave, } \\
\text { Optical fiber }\end{array}$ & $\begin{array}{l}\text { Network associations } \\
\text { for high mobility UE, } \\
\text { wide area coverage }\end{array}$ & $\begin{array}{l}\text { Outdoors; } \\
\text { planned }\end{array}$ \\
\hline Picocell & $23-30 \mathrm{dBm}$ & $\begin{array}{l}\text { Less than } \\
300 \mathrm{~m}\end{array}$ & $\begin{array}{l}\text { LOS microwave, } \\
\text { Optical fiber }\end{array}$ & $\begin{array}{l}\text { Data traffic } \\
\text { offloading from } \\
\text { macrocells, indoor } \\
\text { and outdoor capacity } \\
\text { improvement }\end{array}$ & $\begin{array}{l}\text { Indoors or } \\
\text { outdoors; planned }\end{array}$ \\
\hline Femtocell & $\begin{array}{l}\text { Less than } 23 \\
\mathrm{dBm}\end{array}$ & $\begin{array}{l}\text { Less than } \\
50 \mathrm{~m}\end{array}$ & $\begin{array}{l}\text { Copper cable, } \\
\text { DSL }\end{array}$ & $\begin{array}{l}\text { Indoor coverage area } \\
\text { capacity improvement }\end{array}$ & $\begin{array}{l}\text { Indoors; } \\
\text { unplanned }\end{array}$ \\
\hline Relay & $30 \mathrm{dBm}[7]$ & $300 \mathrm{~m} \mathrm{[7]}$ & Wireless & $\begin{array}{l}\text { Coverage extension, } \\
\text { signal diversity }\end{array}$ & $\begin{array}{l}\text { Indoors or } \\
\text { outdoors; planned }\end{array}$ \\
\hline $\begin{array}{l}\text { RRH/ } \\
\text { DAS }\end{array}$ & $46 \mathrm{dBm}[7]$ & Few kms & Optical fiber & $\begin{array}{l}\text { Cooperative gain, } \\
\text { handoff reduction }\end{array}$ & $\begin{array}{l}\text { Indoors or } \\
\text { outdoors; planned }\end{array}$ \\
\hline $\begin{array}{l}\text { D2D } \\
\text { (UE) }\end{array}$ & $23 \mathrm{dBm}[14]$ & $\begin{array}{l}\text { Short } \\
\text { range }\end{array}$ & Wireless & $\begin{array}{l}\text { Data traffic } \\
\text { offloading from } \\
\text { macrocells }\end{array}$ & $\begin{array}{l}\text { Indoors or } \\
\text { outdoors; } \\
\text { unplanned }\end{array}$ \\
\hline $\begin{array}{l}\text { MTC } \\
\text { (Sensor/ } \\
\text { actuator) }\end{array}$ & $0-20 \mathrm{dBm}$ & $\begin{array}{l}\text { Short } \\
\text { range }\end{array}$ & $\begin{array}{l}\text { Wired or } \\
\text { wireless }\end{array}$ & $\begin{array}{l}\text { Direct } \\
\text { communication } \\
\text { between MTC devices }\end{array}$ & $\begin{array}{l}\text { Indoors or } \\
\text { outdoors; } \\
\text { unplanned }\end{array}$ \\
\hline
\end{tabular}

\subsection{Radio Access Network Performance Enabler}

\subsubsection{Network capacity}

A high system capacity is the major requirement for the evolution of $5 G$ networks. With no specific direction on how the envisaged high capacity of $5 \mathrm{G}$ networks can be achieved, we intend to exploit the Shannon's capacity formula to point out possible ways to enhance the system capacity of $5 \mathrm{G}$ networks. Assume that the link capacity between a UE and a BS is the same as the received throughput at the UE. Using Shannon's capacity formula, the link capacity for a point-to-point communication between a macro BS and a UE considering interference effects at the UE can be expressed as

$$
C_{\text {link }}=B_{\text {link }} \log _{2}(1+P /(I+N))
$$

where $P, I$, and $N$ are the received signal power, the interference power and the additive white Gaussian noise (AWGN) power respectively at the UE. Blink denotes the link bandwidth.

Assume that there are $S$ links that can be provided with the system bandwidth $B_{y y s}$. For simplicity, assume that all UEs have the same link characteristics such that values of $P, I$, and $N$ are the same for all UEs. Hence, 
the system capacity is directly proportional to the link capacity. Consider also that there are more than one antenna at the BS and at the UE such that there exist $M$ parallel spatial channels in a link, where $M=\min \left\{n_{n}, n_{r}\right\}$ denotes the spatial multiplexing gain, and $n_{t}$ and $n_{r}$ denote respectively the number of antennas at the BS and at the UE. Assume that small cells are deployed in the coverage of a macrocell and are operated with the same system bandwidth, i.e., $B_{s y s}$, such that the bandwidth is reused $K$ times to small cells. Hence, using Eq. (1), the system capacity of HetNets for a single macrocell overlaid by small cells can be given as

$$
C_{\text {sys }}=K M B_{\text {sys }} \log _{2}(1+P /(I+N))
$$

Note that we implicitly consider that the received power is the same at a UE irrespective of whether the UE is served by a macrocell or a small cell for simplicity and consistency. The assumption is reasonable enough in a sense that the higher transmission power of a macro BS than a small cell BS is compensated by good channel conditions between a UE and a small cell BS because of lower path loss, higher probability of LOS link, and less fading effect in a small cell than a macrocell. In addition, we assume that the $M$ spatial channels have the same capacity. By analyzing Eq. (2), possible ways to improve each element that eventually contributes to an improvement of the overall system capacity $C_{y y s}$ of $5 \mathrm{G}$ HetNets can be pointed out and are mentioned in the followings:

1) The denser a HetNet, the higher the interference effects through the term $I$.

2) By increasing the density of small cells per unit area, more frequency reuse (i.e., higher $K$ ) can be performed.

3) By implementing a massive number of antennas at a macro BS, a large spatial multiplexing gain $M$ can be achieved. This implementation of a large number of antennas at a BS is called massive MIMO. In addition to massive MIMO, other advanced technologies such as networked MIMO and distributed antenna systems (DAS) can also be used.

4) The more the system bandwidth, the higher the system capacity. Since most frequencies below $3 \mathrm{GHz}$ have almost been utilized, possible ways to increase spectrum bandwidths are to aggregate spectrums from mmWave bands, ranging from 3 to $300 \mathrm{GHz}$. From mmWave spectrum bands, several tens of $\mathrm{GHz}$ of bandwidth would be made available for the capacity of $5 \mathrm{G}$ networks [8].

5) An adaptive power control techniques and cooperative communications (e.g., CoMP, eICIC) can be applied to improve signal power $P$ and to mitigate interference power $I$ at a UE.

In the following subsections, these enabling technologies are discussed.

\subsubsection{Dense HetNets}

Since low power small cell BSs deployed in a macrocell coverage can contribute to improving the system capacity of $5 \mathrm{G}$ networks, the capacity gain can be improved further if the system bandwidth can be reused as many times as possible. A reuse of the system bandwidth depends mainly on two aspects, namely, deployment scenarios and small cell densities, and is done by the co-channel deployment of small cells within a macrocell. The reuse can be increased significantly by a dense deployment of small cells per unit area. There have already been tremendous consideration on network densification toward 5G networks. A rich amount of researches have addressed many issues of dense HetNets, for example, a general overview on dense HetNets [38], the role of small cells [39], performances of dense HetNets from various deployment perspectives [40], coordination of interferences in dense HetNets [41], cooperative distributed radio resource management in hyper-dense HetNets [42], energy efficiency, spectral efficiency and QoS trade-off [43], coverage analysis of LTE urban HetNets with dense femtocells [44], and a novel architecture using small cells to address MTC traffic [45].

In dense HetNets, an extremely large number of small cells are deployed where the coverages of small cells can even overlap one another. However, this raises the concern of generating significant interferences from one cell to another. A straightforward way to address the interference effects is to allocate orthogonal frequencies to small cells. However, this approach directly impacts a limited and highly expensive system bandwidth, and hence the achievable system capacity. Another approach is to ensure a very tight coordination among cells such that the same frequency can be reused among small cells with an appropriate cooperation. The use of cooperative principles will play a significant role on the $5 \mathrm{G}$ network capacity achievement. We discuss cooperative communications next. 


\subsubsection{Cooperative communications}

Cooperation can be defined as a process of working together [46]. In cellular wireless networking aspects, a number of BSs and UEs cooperate to address common network goals such as channel diversification, resource allocation, interference mitigation, and so on. The perspectives of cooperation are broad, and in this paper, we particularly emphasize those perspectives that are highly relevant on current mobile networks, specifically the 3GPP LTE with a projection toward future $5 \mathrm{G}$ networks. The concept of cooperative communications was addressed first in [47], where the author proposed a three-terminal relay channel with a derivation of upper and lower limits of capacity. Later, the authors in [48] investigated the capacity of a cooperative relay channel and set a theoretical basis for the research on cooperative communications. Currently, in LTE release 10 (LTEAdvanced), cooperative communications has been standardized as one of the advanced technologies to address many crucial issues such as interference, capacity, diversity, cell-edge user throughput by including relay stations (RSs) and cooperative multi-point (CoMP) communications. RSs are used to allow communication to a destination node by relaying signals from the network and vice versa. In CoMP, a group of network nodes (also termed a cooperating set) coordinate among each other, and this type of cooperation is termed as node cooperative systems.

RSs use three possible cooperative protocols, namely, amplify-and-forward, decode-and-forward, and compress-and-forward. If a backhaul link is poor, amplify-and-forward and compress-and-forward protocols are favorable. However, for a good relay backhaul link, decode-and-forward is more advantageous [49]. Since relays are deployed in the coverage of a macrocell, relay cooperative schemes are also called intra-cell CoMP where RSs and a macro BS cooperate. Cooperation in node cooperative systems can be held by either joint processing $(\mathrm{JP})$ or coordinating strategies among nodes in a cooperating set. In JP, data among all cooperating nodes are first exchanged via backhaul links, and transmissions and receptions of data take place jointly from all nodes at a time to improve mainly user throughputs. All cooperating nodes are connected to each other via high speed backhaul links, and are forming a DAS which can easily take advantage of spatial diversity, resulting in an improved overall network capacity. In the coordinated node cooperation, all cooperating nodes in a set coordinate strategies, e.g., resource allocation, beamforming pattern via backhaul links to reduce interferences from adjacent nodes. Control information such as channel state information (CSI) is shared for coordination among all cooperating nodes.

JP is further categorized into two, namely, joint transmission (JT) and dynamic point selection (DPS). Irrespective of CoMP scheme, control signals, e.g., physical downlink control channel (PDCCH) are only transmitted from the serving cell, i.e., the cell where the current physical location of a UE exists. In JT, all points or partly in a cooperating set are associated with a UE specific demodulation reference signal (US-RS) and transmit user data simultaneously to a UE coherently or non-coherently in a time-frequency resource to improve received signal qualities and data throughputs of the UE. However, in DPS (muting), user data are transmitted from only one point of a cooperating set, while all other points are muted even though the user data is available at all points. Transmitting or muting a point may change simultaneously from one subframe to another following a specific scheduling strategy such as the minimum path loss between a point and a UE [50].

In coordinated scheduling/coordinated beamforming (CS/CB), user data are available only in the serving point, however scheduling or beamforming decisions are taken after coordinating with all other nodes in a cooperating set. With a combination of JP and CS/CB that results in a hybrid scheme, a few points can involve in JT simultaneously, while other points can cooperate for CS/CB in a time-frequency resource. All these schemes are shown in Fig. 3. CoMP communications have been considered for LTE Release 11 [51], and a number of scenarios of CoMP communications are considered for both uplink and downlink in HomNets as well as HetNets [52].

\subsubsection{Cooperative interference management}

Interference is one of the major bottlenecks and is more severe in dense HetNets than in traditional HomNets. The major sources of interference in HetNets are unplanned deployment, restricted femtocell access, transmission power difference among nodes, and new techniques such as cell range expansion (CRE) [7]. 
Femtocells are partly deployed by users in an unplanned fashion with almost no consciousness on density, location, and access type of femtocells from an operator perspective. If the access type of a femtocell is configured as CSG, interference effects become the most severe in comparison with other access types. More specifically, in co-channel deployment of a femto BS, a macro UE close to the coverage of a CSG femto BS is interfered highly by the CSG femto BS in its downlink reception as shown in Fig. 4(a) as the macro UE is not allowed to get access to the femto BS. Similarly, a macro UE at far distance (e.g., near cell-edges) from a macro BS transmits in uplink at a high power to compensate the path loss, and hence originates cross-tier interferences by jamming uplink transmissions from femto UEs to a femto BS. Further, the unevenness in transmission powers of different nodes in HetNets is another potential cause of interference. Since a UE usually gets connected to a BS with the highest downlink signal strength in its neighbor BSs, a UE prefers to get connected with a macro BS because of its higher transmission power than that of a small cell BS, e.g., pico BS. This creates a phenomenon called imbalance in load distribution between cells, and a macro BS is likely to get overloaded almost always even though there are pico BSs around UEs at much shorter distances than those from the macro BS. Moreover, UEs in the near coverage of a pico BS is interfered highly in downlink from the pico BS (Fig. 4(b)). If a UE near a pico BS is connected to the pico BS, this can help the UE not to suffer from downlink interference since the UE is now communicating with the pico BS and also offloading traffic from the macro BS.

Both offloading problems and downlink interferences for a macro UE can be addressed by expanding the actual picocell area by adding an offset to the reference signal received power (RSRP) of a pico BS which is referred to as cell range expansion (CRE) [53]. When a pico BS is employed with CRE, a UE receives a higher RSRP from the pico BS, and hence more UEs are likely to get connected to the pico BS. This results in more macro UEs to be offloaded to the pico BS from a macro BS and less downlink interferences at a macro UE. However, those pico UEs which are within the expanded cell area of a pico BS are likely to suffer from downlink interferences caused by a macro BS as shown in Fig. 4(c). This can be overcome by coordinating resource scheduling decisions between a macro BS and a pico BS via backhaul links. For example, by scheduling orthogonal resource blocks (RBs) by a macro BS to macro UEs near the victim pico UEs.

D2D communication links also suffer from both intra-cell and inter-cell interferences. The intra-cell interference occurs mainly from a D2D UE to a macro UE when both types of UE are assigned the same resources. The inter-cell interference occurs between a D2D UE and nearby macro BSs around the serving BS that serves the D2D UE. With a coordination among macro-BSs, the inter-cell interference at the D2D UE, particularly at the cell-edge area, can be overcome by exchanging resource scheduling and allocation decisions via backhaul links between macro BSs. The intra-cell interference for a D2D UE can be overcome by the serving macro BS using well-known algorithms such as zero-forcing (ZF) and minimum mean-square-error (MMSE) based on the CSI received from an affected UE by employing appropriate precoder matrices for the affected UE. Figure 4(d) [37] shows both inter-cell and intra-cell interferences in D2D communications.

Interferences in HetNets can better be managed by using frequency domain, time domain and power control inter-cell interference coordination (ICIC) techniques which are aggregately called as enhanced ICIC (eICIC) [7]. In time domain eICIC techniques, a victim UE is scheduled in time domain while interferences from other nodes are reduced. The use of almost blank subframe (ABS) is one way to address time domain eICIC techniques where the victim UE is scheduled during ABSs [7]. As mentioned earlier, interferences from a CSG femto BSs can be overcome by using ABS based eICIC. For example, if a macro UE is near to the coverage of a CSG femto BS, ABSs can be applied at the femto BS such that the macro UE will be scheduled only during ABSs. In an ABS, data and control signals are not scheduled; however, only reference signals are scheduled. Another approach to address time domain eICIC is to apply shifting to the subframe boundary by a number of orthogonal frequency division multiplexing (OFDM) symbols of one BS with respect to the other such that control channels of both BSs do not overlap each other. However, control channels of the macro UE (near the femto BS) are still interfered by data channels of femto UEs. To overcome this problem, one approach is to mute those overlapped OFDM symbols of the femto BS with the control channels of the victim macro UE. The other approach is to configure subframes of the femto BS as ABSs that overlap control channels of the macro UE. 


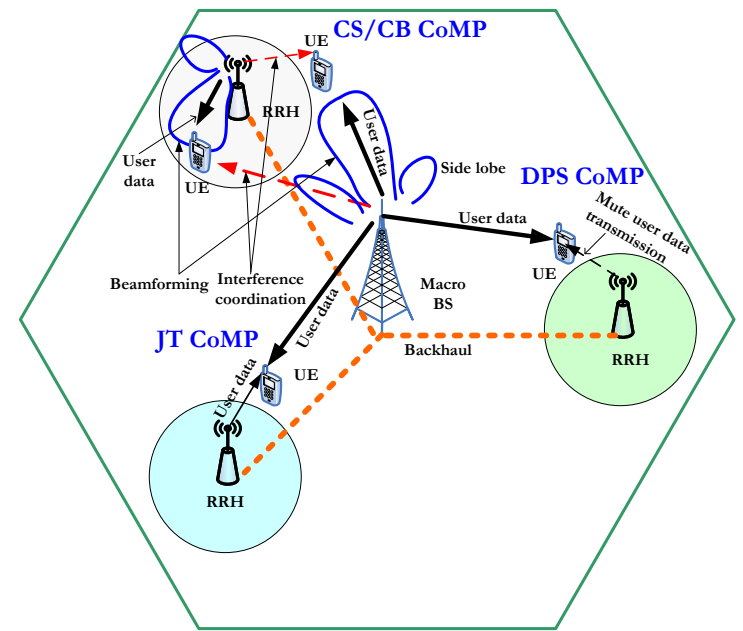

Fig. 3. CoMP schemes in HetNets.

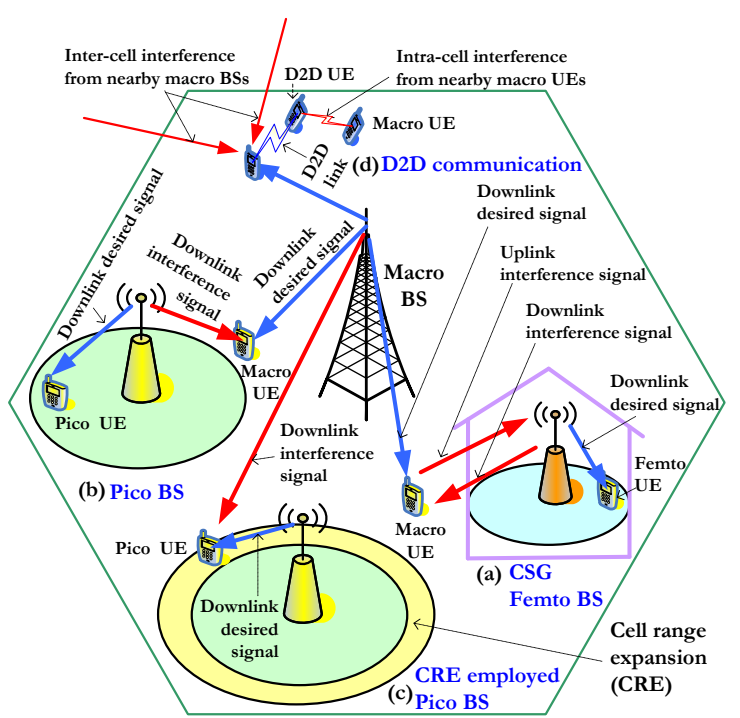

Fig. 4. Interferences in HetNets.

In frequency domain eICIC techniques [7], by orthogonal scheduling of control channels between cells in a reduced bandwidth, interferences can be mitigated. Along with a static orthogonalization, a dynamic frequency domain orthogonalization can be performed by detecting a victim UE. An interfered UE can be detected at a BS based on the UE specific measurement reports. The victim UE's BS then informs the interferer BS via backhaul signaling. A victim UE can also be detected by an interferer BS and can coordinate its scheduling decision with the victim UE's BS [7]. In power control eICIC techniques, different power control techniques can be applied to small cells [7]. However, a reduction in transmission power at a femto BS will eventually reduce throughputs of its femto UEs at the gain of reduced interference for macro UEs. Some trade-offs can be considered on scenarios of interest such that an optimal power control at small cells can be adopted and may contribute to an overall system capacity improvement.

\subsubsection{Cooperative multi-antenna systems}

Employing multiple antennas at both a transmitter and a receiver is one of the most useful technologies to increase spectral efficiency and system capacity, and literally the configuration is called MIMO. To avoid ambiguity, we refer to a BS as transmitter and a UE as receiver in the downlink and in the uplink a UE as transmitter and a BS as receiver in a cellular system. At the system level, the major concepts of multiple-antenna system implementation are either centralized or distributed. In the centralized concept, a number of antennas are collocated at the same BS with a few wavelengths apart from one another. Centralized systems usually work best in terms of performance improvements in capacity and diversity when channels, observed by each antenna, are highly scattered such that the correlation between channels from an antenna array system is the least or none at all. However in reality, because of implementation specific constraints (e.g., physical limitations at transceivers), a large number of antennas are implemented within a limited space. This causes a high correlation between channels and a low degree of freedom which results in a poor performance. However, if the same number of antennas are distributed geographically and are jointly processed at a central station via ideal backhaul links such as optical fibers, a high degree of independent channels can be achieved [54]. Because of spatial diversities, channel characteristics from these antennas varies significantly from one to another at the same UE and hence results in an improved capacity from achieving a high received signal diversity gain. Further, a reduction in transmission power and path loss can be achieved from spatial diversities of these distributed antennas. This type of multi-antenna system is called DAS [54] and is illustrated in Fig. 5(a).

In MIMO systems, when there is only one user in a BS coverage, we refer to the scenario as a single userMIMO (SU-MIMO) system as shown in Fig. 5(b). An SU-MIMO system suffers from a high channel correlation since multiple antennas are spaced apart by a short distance both at a BS and a UE. Further, the 
capacity of a SU-MIMO system is limited by the number of antennas of a UE. This is because the capacity of a MIMO system varies proportionally with spatial multiplexing gain of the link between a BS and a UE, where the gain is directly proportional to the lesser between the numbers of antennas of a transmitter (BS) and a receiver (UE) in downlink, and a UE usually has fewer antennas than a BS. To overcome this problem, a high diversity in spatial channels needs to be achieved. One way to do this is to employ MIMO principles to more than one UE by exploiting randomness of UE distributions in the coverage of a BS, and the resulting system is called multi-user-MIMO (MU-MIMO) as shown in Fig. 5(c). However, the inter-cell interference experienced by UEs, particularly cell-edge UEs, from nearby BSs and UEs is the major bottleneck to an improvement in the overall system capacity.

A cooperation between nearby BSs can be exploited to keep this interference at a minimal or zero level. By introducing a coordination between BSs, a higher degree of freedom can be achieved. This configuration is called networked MIMO where a group of BSs coordinate with each other to form a virtual massive multiantenna system for downlink transmission as shown in Fig. 5(d). In a networked MIMO system [55], data streams from multiple BSs are simultaneously transmitted to multiple UEs within or beyond their cell coverages by cancelling cross-talk interferences. This results in achieving a spatial multiplexing gain that scales system capacity with cluster size (i.e., the density of cooperating BSs). However, it requires a tight synchronization in terms of transmission time, carrier frequency, sampling clock-rate, and sharing of user data between cooperating BSs for cancelling cross-talk interferences. Since overheads from cooperating BSs increase with cluster size, a networked MIMO system is feasible for small networks. As proposed in [56], CoMPs with MUMIMO can also be exploited to improve capacity by taking advantages from both the spatial multiplexing gain of MU-MIMO systems and the interference avoidance (nullification) of CoMP systems.

However, 5G networks are expected to support 1000 times the volume of data as the current networks do. In order to address the high capacity demand of $5 \mathrm{G}$ networks, a large number of antennas, e.g., hundreds, are expected to be deployed in $5 \mathrm{G}$ networks. This antenna configuration is called massive MIMO or large antenna systems as shown in Fig. 5(e). A massive MIMO system relies on a high spatial multiplexing gain, and hence it is assumed that a BS has channel knowledge of both uplink and downlink. In uplink, UEs can send pilots, and a BS can measure the channel response of each UE based on these pilot signals. However, downlink channel measurements are not easy. In an frequency division duplex (FDD) based current LTE systems, BSs send pilots to UEs; UEs then estimate channels based on pilots; and after quantizing estimated channels, UEs send them back to BSs [57]. However, this process is challenging in the case of a massive MIMO system, particularly for high-mobility users, mainly for two reasons. Firstly, it requires 100 times more resources in downlink than in conventional systems to ensure mutually orthogonal pilots between antennas. Secondly, there is a proportional increase in the number of channel responses with BS antennas for uplink. That is why massive MIMO systems are expected to be operated on a time division duplex (TDD) mode where it can use channel reciprocity between uplink and downlink [57]. A number of representative features and challenges of massive MIMO systems are discussed in the followings.

Features of massive MIMO systems:

1) Operation: In massive MIMO systems, multiple data streams are sent using the spatial-division multiplexing (SDM) technique such that different data streams can use the same time and frequency resources. The SDM is performed with a large number of independently-controlled antennas. Data streams are precoded before transmitting based on the channel responses which are sent in the uplink by UEs and received at each antenna of the antenna-array. The precoding matrix is chosen such that the desired signal is strong enough as compared to the interference and noise signals at each intended UE [58]. Similarly, in the uplink, each UE transmits its data stream using the same time and frequency resources. The sum data from UEs is received at each antenna and then decoded to retrieve the received signals to produce individual data streams.

2) Architecture: Massive MIMO systems can be deployed using an array of antennas which are either colocated at the same BS or distributed to cover a certain area [59]. Further, a macro BS can be replaced by a large number of low power antennas. 
3) Scalability: Massive MIMO ensures the scalability issue by employing the time division duplex (TDD) method. In contrast to the frequency division duplex (FDD) method, TDD is used because of the fact that the time required to collect the channel responses is independent of the number of antennas [58], and there is almost no bound on the maximum number of antennas that is to be considered in massive MIMO systems with TDD [8].

Challenges of massive MIMO systems:

1) Channel estimation: Estimating the channel responses accurately is critical to update the precoding matrix, and is one of the major limitations of massive MIMO systems [8]. This is because of the fact that the UE velocity puts a limit on the time interval during which the channel response must be acquired. This in turn limits the maximum number orthogonal pilots that can be obtained and the maximum number of UEs that can be served simultaneously [58].

2) Pilot contamination: Because of the limited number of pilots for channel estimations, pilots are to be reused in nearby cells. Hence, the pilot sequence of one cell to serve its UEs can be interfered by another cell that uses the same pilot sequence to serve its UEs. This phenomenon is called pilot contamination [58] [60], and is another major challenges for the massive MIMO system implementation.

3) Economic and standardization impacts: The cost effectiveness of developing massive MIMO systems is not explicitly justified yet [8]. In addition, the real implementation of massive MIMO systems may require a new standard [58].

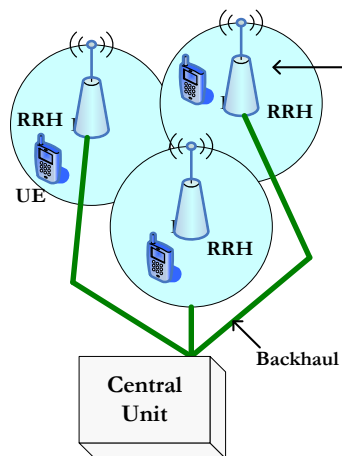

(a) DAS

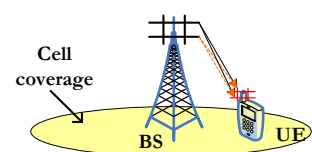

(b) SU-MIMO

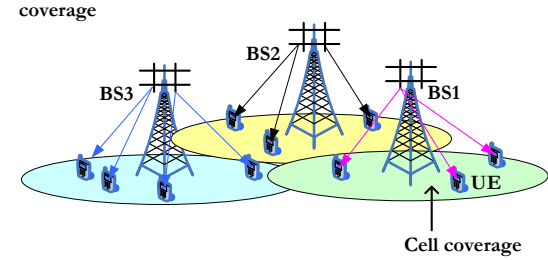

(c) MU-MIMO

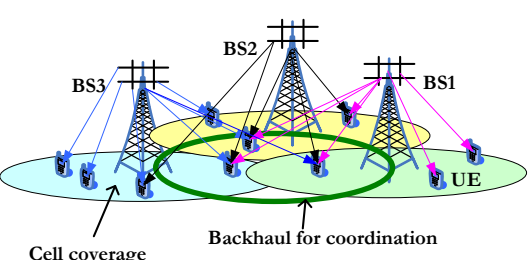

(d) Coordinated MU-MIMO

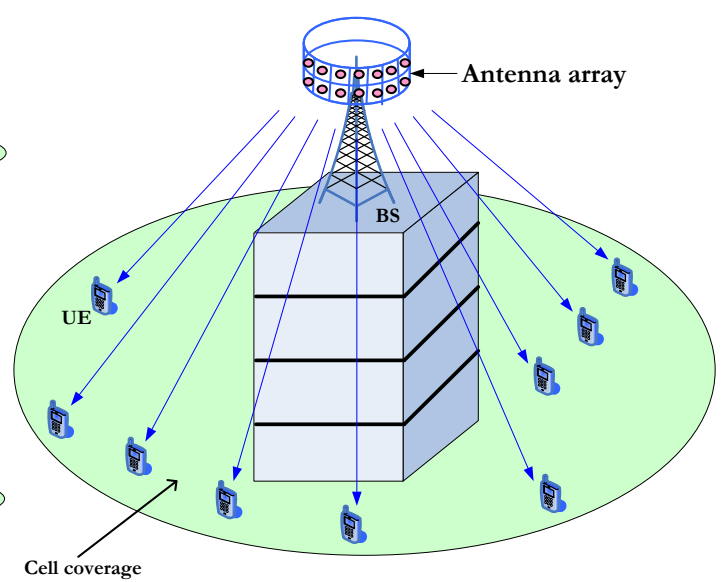

(e) Massive MIMO

Fig. 5. Multi-antenna configurations.

\subsubsection{Millimeter wave communications}

Incentives and features of mmWave: One of the major enablers to address the high traffic demands of $5 \mathrm{G}$ networks is to increase system bandwidth by using spectrum aggregation techniques. Current microwave frequencies ranging from $700 \mathrm{MHz}$ to $2.6 \mathrm{GHz}$ [61] for cellular wireless are almost saturated by their practices. Currently, the global bandwidth allocation for cellular wireless is limited to $780 \mathrm{MHz}$, and an approximate maximum bandwidth of $200 \mathrm{MHz}$ for each major wireless provider across different bands [61-62]. This motivates the use of millimeter wave (mmWave) spectrums for $5 \mathrm{G}$ networks. According to [61], one of the major differences of $5 \mathrm{G}$ systems from $4 \mathrm{G}$ systems will be in the use of greater spectrum allocations at mmWave bands. In addition, backhaul networks will be implemented at mmWave bands instead, by replacing existing copper and fiber 
backhauls. The implementation of a high gain and steerable antenna both at a UE and a BS makes mmWave communications viable over the wireless medium [61, 63-64]. Noticeable features of mmWave [61] that make the movement toward mmWave communications are as follows:

1) An availability of a large amount of usable spectrums in mmWave bands results in achieving a high data rate.

2) An expansion of channel bandwidth beyond $20 \mathrm{MHz}$ [65].

3) A small wavelength of mmWave results in an exploitation of new spatial processing techniques such as massive MIMO, adaptive beamforming [66].

4) An availability of a large amount of bandwidth allows handling capacity for BS-to-UE link and BS-toBS backhaul link in highly populated areas greater than the current 4G systems

5) Wireless features result in flexibility and quick deployment of backhauls in dense urban areas.

6) A small coverage by mmWave results in an exploitation of spatial frequency reuse techniques and new cooperative techniques such as networked MIMO, cooperative relays.

Propagation characteristics of mmWave: MmWave spectrums have a number of essential propagation characteristics that make the mmWave difficult to achieve seamless coverage and reliability [67-69]. Firstly, an mmWave signal experiences a much higher propagation loss than a microwave signal, which can be compensated by a high-gain directional antenna. Secondly, an mmWave signal faces difficulty in diffracting around obstacles because of its small wavelength. Further, LOS propagations can be blocked by obstacles, and non-LOS can be blocked from a shortage of multiple paths and a significant attenuation. All these result in a possibility for a link outage if a LOS path does not exist. Thirdly, an mmWave propagation faces difficulty from penetrating solid materials (e.g., a $178 \mathrm{~dB}$ attenuation at $40 \mathrm{GHz}$ for brick walls [67]).

Spectrums of mmWave: MmWave spectrums range from $3 \mathrm{GHz}$ to $300 \mathrm{GHz}$ with corresponding wavelengths range from $1 \mathrm{~mm}$ to $100 \mathrm{~mm}$ [70]. However, from this range of mmWave spectrums, the oxygen absorption band which ranges from $57 \mathrm{GHz}$ to $64 \mathrm{GHz}$ with an attenuation about $15 \mathrm{~dB} / \mathrm{km}$, and the water vapor absorption band which ranges from $164 \mathrm{GHz}$ to $200 \mathrm{GHz}$ with an attenuation about tens of dBs are to be excluded for mmWave communications. Assume that 40 percentage of the remaining $252 \mathrm{GHz}$ can be potentially explored for the use of mmWave communications, then a possible new $100 \mathrm{GHz}$ of spectrum [7071] can be exploited for $5 \mathrm{G}$ mobile communications. In an announcement by the Federal Communications Commissions (FCC) in October 2003, 71-76 GHz, 81-86 GHz, and 92-95 GHz frequencies that constitute aggregately a total of $12.9 \mathrm{GHz}$ bandwidth became available for ultra-high-speed-data communications [70].

Further, the licensed $28 \mathrm{GHz}$ and $38 \mathrm{GHz}$ bands have an available bandwidth of over $1 \mathrm{GHz}$ that could be used for mobile access and backhaul networks $[61,72]$. Even though rain and atmospheric attenuations are the major unavoidable drawbacks for mmWave spectrums, the impact from them is not significant enough for 28 $\mathrm{GHz}$ and $38 \mathrm{GHz}$ bands because of small mmWave cell sizes. There is only a $1.4 \mathrm{~dB}$ attenuation from a heavy rain fall over $200 \mathrm{~m}$ distance at $28 \mathrm{GHz}$. For small distances, typically less than $1 \mathrm{~km}$, the rain attenuation has a minimal effect on mmWave propagations at $28 \mathrm{GHz}$ and $38 \mathrm{GHz}[61,73]$. Further, for $28 \mathrm{GHz}$ and $38 \mathrm{GHz}$, the atmospheric absorption is insignificant for an mmWave cell size of $200 \mathrm{~m}$.

Access link and backhaul link of mmWave: $\mathrm{MmW}$ ave technologies have been proposed for $5 \mathrm{G}$ mobile networks [74]. MmWave BS and backhaul systems are expected to be deployed on urban utility poles, street lamps, and building frontages. Access links of mmWave will be assisted by a macro BS for control signaling and synchronization purposes, while user data at a high speed will be provided to a UE by an mmWave BS. Hence, C-plane will be served by a macro BS and U-plane will be served by an mmWave BS. With a multi-hop short distance (up to $100 \mathrm{~m}-200 \mathrm{~m}$ ) LOS mmWave backhaul, a peak capacity of 10-25 Gbps can be provided, and the backhaul can be extended up to $1 \mathrm{~km}$. For such a long distance backhaul, high-end solutions with highly directive antennas (e.g., $30 \mathrm{dBi}$ to $52 \mathrm{dBi}$ gain) are emerging at E-bands [75-76]. Data rates up to $2.5 \mathrm{Gbps}$ using 64 quadrature amplitude modulation (QAM) on a $500 \mathrm{MHz}$ wide channel has already been announced [76].

Moreover, mmWave backhauls are now commonly accepted among the main network equipment providers, and several prototype network implementations for measurement campaigns have been carried out, e.g., Nokia Siemens Networks in New York at $72 \mathrm{GHz}$, Samsung in New York, Austin, and Korea at $28 \mathrm{GHz}$ and $38 \mathrm{GHz}$ [76]. However, the main constraints for access link come from UE requirements. For example, UEs are equipped with multi-radio support capabilities such as WiFi and GPS, each operating at a distinct 
frequency with multiple antennas. Further, there is a disproportionate advancement in the development of UE battery technologies with applications. All these make mmWave a technology to be embedded at a minimal impact on the existing hardware of a UE with a minimal space for setting up of a new mmWave radio and antenna. Furthermore, a mmWave access link needs to communicate with a larger coverage in outdoor environments than in indoor environments. This larger coverage in outdoor can be addressed with a high gain antenna and a high transmission power where a UE's size and power consumption can be relaxed [76].

Applications of mmWave to emerging technologies: MmWave wavelengths are attractive for new emerging technologies such as massive MIMO to enhance spectral efficiency since more antennas can be located with a reduced antenna array size. A large gain from beamforming with a large number of antennas can supplement high path losses of mmWave spectrums. However, cell discoveries in mmWave face problems from the directivity. To overcome this problem, small cell discoveries at mmWave can be done in a number of ways [77]. One way is that a macro BS handles the cell discovery process. In this case, a macro BS knows a small cell coverage and a UE location. The macro BS informs a small cell as a UE heads toward the small cell such that the small cell can steer its beam toward the UE. The macro BS then informs the UE so that the UE can also steer its antenna beam toward the small cell BS. The other way would be to implement both a small cell BS and a UE with a capability to operate on both below $3 \mathrm{GHz}$ and $\mathrm{mmWave}$ frequencies. In this case, cell discoveries are provided at a frequency below $3 \mathrm{GHz}$. Once a UE gets connected to a small cell, the small cell can transmit user data at an mmWave frequency by steering its antenna beam toward the UE. MmWave spectrums can also be applied to new emerging technologies such as D2D communications. There are two kinds of D2D communications that can be enabled in mmWave spectrums, i.e., local D2D and global D2D communications. In a local D2D communication, two devices communicate to one another via the same BS or via a relay node if a LOS signal is present between them. However, in a global D2D communication, two devices are typically associated with two different BSs and can communicate to one another via mmWave backhauls [67].

Further, to address the high capacity demand of $5 \mathrm{G}$ networks, small cells are expected to be deployed densely. But, a dense deployment of small cells raises several issues. One of the major concerns is a high cost of fiber backhauls for every small cell which in turn raises the practicability of deployment of backhauls to small cells using fibers. However, using wireless backhauls at microwave frequencies to reduce cost is already a wellaccepted approach, and an extensive number of microwave point-to-point links are in operation in $4 \mathrm{G}$ systems [78-82]. Authors in [78] have proposed a point-to-multipoint inband mmWave wireless solution where both access and backhaul links are multiplexed on the same frequency to address low cost and low latency requirements of ultra-dense $5 \mathrm{G}$ networks. Note that usually out-of-band backhauls are dominant in wireless industry [81-82] to overcome an extreme capacity demand in licensed access frequency bands [83-84]. However, in mmWave backhauls, a channel bandwidth can be large so that inband backhaul approach can be deployed [78]. In Table 3, a comparative analysis for network capacity improvement approaches, i.e., cooperative communications, multi-antenna systems, dense HetNets, and mmWave communications is given.

\section{Network Control Programming Platform}

The programmable feature of network control is one of the most impactful aspects toward the evolution of $5 \mathrm{G}$ networks. The traditional way of controlling wireless networks is based on programming networks in a distributed manner where control plane is distributed network wide both logically and physically in networking devices. However, the application of SDN concepts to wireless networks allows controlling networks logically in a centralized software platform. Usually, wireless network control mechanisms are based on either a local or a global network view. In a distributed programmable network, typically a network control mechanism is based on a local network view. The local network view is usually centered on a network entity such as a cell and a UE where the former is termed as cell-centric, and the latter is termed as device-centric network control. On the other hand, in a centralized programmable network, a network control mechanism is usually based on a global network view where a centralized network entity controls the whole network with a global information of the network. This centralized network entity is termed literally as controller which can be implemented physically in a distributed manner where physical controllers exchange information to one another, but logically they can act as a single control entity [85-86]. Indeed, several aspects such as scalability, reliability, and satisfactory 
performance would require a logically centralized controller be physically distributed rather than implementing with a single physical entity [85]. Moreover, a production level SDN design would rather go for a physically distributed control plane [85-86]. 5G networks can be evolved in either a logically centralized or a logically distributed programmable control networks, and we discuss each of these approaches in the following sections.

Table 3. A comparative analysis for network capacity improvement approaches.

\begin{tabular}{|c|c|c|c|c|}
\hline Features & $\begin{array}{c}\text { Cooperative } \\
\text { communications }\end{array}$ & $\begin{array}{c}\text { Multiple } \\
\text { antenna systems }\end{array}$ & $\begin{array}{c}\text { mmWave } \\
\text { communications }\end{array}$ & $\begin{array}{c}\text { Dense } \\
\text { HetNets }\end{array}$ \\
\hline Enablers & CoMP, eICIC & $\begin{array}{l}\text { Massive MIMO, } \\
\text { networked MIMO }\end{array}$ & mmWave spectrums & Small cells \\
\hline Objectives & $\begin{array}{l}\text { Interference } \\
\text { management }\end{array}$ & $\begin{array}{l}\text { Spatial multiplexing, } \\
\text { spatial diversity, and } \\
\text { beamforming }\end{array}$ & $\begin{array}{l}\text { Spectrum } \\
\text { aggregations }\end{array}$ & Frequency reuse \\
\hline Improvement & $\begin{array}{l}\text { Spectral efficiency } \\
\text { improvement }\end{array}$ & $\begin{array}{l}\text { Spectral efficiency } \\
\text { improvement }\end{array}$ & $\begin{array}{l}\text { Bandwidth } \\
\text { extension }\end{array}$ & $\begin{array}{l}\text { Capacity } \\
\text { improvement }\end{array}$ \\
\hline $\begin{array}{l}\text { Taking } \\
\text { advantages } \\
\text { from }\end{array}$ & $\begin{array}{l}\text { Coordination } \\
\text { among spatially } \\
\text { separated BSs }\end{array}$ & $\begin{array}{l}\text { An enormous amount } \\
\text { of antennas that can be } \\
\text { implemented on a BS } \\
\text { either in centralized or } \\
\text { distributed ways }\end{array}$ & $\begin{array}{l}\text { An enormous } \\
\text { amount of available } \\
\text { bandwidth }\end{array}$ & $\begin{array}{l}\text { Short distance } \\
\text { between a UE and } \\
\text { a BS }\end{array}$ \\
\hline Requirements & Strict backhaul & $\begin{array}{l}\text { Maximization of the } \\
\text { minimum between the } \\
\text { numbers of transmit } \\
\text { and receive antennas }\end{array}$ & $\begin{array}{l}\text { Small coverage area } \\
\text { typically with } 200 \mathrm{~m} \\
\text { cell radius }\end{array}$ & $\begin{array}{l}\text { Maximization of } \\
\text { frequency reuse }\end{array}$ \\
\hline Methodologies & $\begin{array}{l}\text { By exchanging CSI } \\
\text { and information of } \\
\text { data among BSs }\end{array}$ & $\begin{array}{l}\text { By creating multiple } \\
\text { parallel data streams } \\
\text { and beamforming } \\
\text { between a BS and a UE }\end{array}$ & $\begin{array}{l}\text { By enabling highly } \\
\text { directive LOS link } \\
\text { between a UE and a } \\
\text { BS in a short range }\end{array}$ & $\begin{array}{l}\text { By deploying small } \\
\text { cells as densely as } \\
\text { possible in a } \\
\text { macrocell coverage }\end{array}$ \\
\hline $\begin{array}{l}\text { Enabling } \\
\text { degrees-of- } \\
\text { freedom }\end{array}$ & $\begin{array}{l}\text { CoMP (JP, DPS, } \\
\text { and CS/CB) and } \\
\text { eICIC (time } \\
\text { domain, frequency } \\
\text { domain, and power } \\
\text { control techniques) }\end{array}$ & $\begin{array}{l}\text { Number of antennas } \\
\text { for a massive MIMO } \\
\text { system and cluster size } \\
\text { for a networked MIMO } \\
\text { system }\end{array}$ & $\begin{array}{l}28 \mathrm{GHz}, 38 \mathrm{GHz}, \\
60 \mathrm{GHz}, 71-76 \\
\mathrm{GHz}, 81-86 \mathrm{GHz}, \\
\text { and } 92-95 \mathrm{GHz}\end{array}$ & $\begin{array}{l}\text { Picocell, femtocell, } \\
\text { RRH, and relay }\end{array}$ \\
\hline Appl & $\begin{array}{l}\text { Macrocell and } \\
\text { small cells }\end{array}$ & $\begin{array}{l}\text { Macrocell and small } \\
\text { cells }\end{array}$ & $\begin{array}{l}\text { Backhaul and small } \\
\text { cells }\end{array}$ & cells \\
\hline
\end{tabular}

\subsection{Logically Distributed Programmable Control Network Platform}

\subsubsection{Cell-centric control networks}

In traditional cell-centric architectures, a UE gets services from a network by making a connection establishment both in uplink and in downlink that carry user data traffic and control signals by using either an FDD or a TDD technique. All BSs (typically macro BSs) have usually the same transmission power for HomNets. BSs are deployed and scaled on capacity demand, following a cell planning strategy, e.g., frequency resource reuse factor. A network is controlled in a distributed manner where each BS takes responsibilities of all UEs under its coverage and hands off to an adjacent BS with the assistance of a mobility management entity (MME). Functionalities of all layers (i.e., layer 1, layer 2 and layer 3) are performed at a macro BS. All UEs within a macro BS coverage are under the sole control of BS. User-plane (U-plane) and control-plane (C-plane) functionalities of a UE are performed locally at a macro BS. A UE is controlled by the network employing both C-plane and U-plane connectivity which are governed by the same entity. For example, when a UE initiates a 
service request to a macro BS, from the network and cell selection procedures to an end of a service session, all control signaling and user data transferred between the macro BS and the UE over the whole session are governed by the same macro BS as long as the UE does not reselect or hand-off to a different macro BS.

After successfully handing off, only a new macro BS governs the UE, and all functionalities regarding control and data planes are transferred to the new macro BS from the old macro BS. In short, a UE cannot be served for C-plane and U-plane functionalities by different BSs at a time. To boost capacity of HomNets, small cells can be deployed in the coverage of a macrocell such that the same carrier can be reused in the small cell tier.

\subsubsection{Device-centric control networks}

In a device-centric control network architecture, a device should be able to communicate with a network through multiple nodes by exchanging multiple flows in a HetNet [8]. Hence, a set of nodes provide connectivity to a device, and functions of these nodes should be contextualized to a specific device during a session. To introduce a device specific architecture to $5 \mathrm{G}$ networks, many changes on existing cell-centric networks need to be addressed, e.g., decoupling C-plane and U-plane, serving uplink and downlink by separate nodes, decoupling baseband processing unit from processing hardware unit of a node, cooperative communication between nodes, redefinition of network architectures for an ad-hoc type communication, and beamforming with a high antenna directivity at mmWave spectrums. A model of device-centric network architecture for 5G networks is shown in Fig. 6 [8]. Because 5G networks will encompass heterogeneous nodes, many of which will likely be unplanned by the network provider, flexible and reliable accesses of these nodes to the network will become crucial issues. Decoupling features have been proposed to address these issues. In the following, a number of proposed decoupling features are discussed.

Decoupling C-plane and U-plane: In distributed HetNets, small cells can be deployed with a separation of Cplane and U-plane. In this configuration, U-plane of a UE is served by small cells, whereas C-plane is served by a macrocell. A small cell simply provides U-plane traffic, and the macrocell provides C-plane traffic to a UE. Small cells are not configured with cell-specific signals, e.g., synchronization signals, and all radio resource control (RRC) connection procedures are provided by the macrocell. Hence, these small cells are also termed as Phantom Cells [87]. Small cells can be assigned with a high frequency band such as mmWave. However, to provide the mobility requirement, the macrocell is assigned with an existing microwave cellular spectrum. This can help address a high capacity need for $5 \mathrm{G}$ networks by small cells, and at the same time, reduce the number of hand-offs because of large coverage area by the macrocell. There are two scenarios for realization of C-plane and U-plane splitting, namely, small cells with baseband processing located at a macro BS and small cells with independent baseband processing. In scenario 1, all baseband processing of small cells and a macrocell are performed at the macro BS. Small cells simply carry U-plane traffic to a UE. Though physically separated, the macro BS and a small cell BS are logically seen as a single entity. Because of common U-plane processing of all small cells, there is a limitation to the maximum number of small cells that can be deployed in a macrocell coverage. This scenario can be applied to the 3GPP LTE carrier aggregation deployments [88].

In scenario 2, each small cell has its independent baseband processing system, and hence a small cell appears as a separate entity from the macrocell. This configuration of C-plane and U-plane overcomes the limitation of scenario 1 because of a local baseband processing at a small cell itself and helps scale network capacity. Based on scenario 2, a new radio access network architecture that employs C-/U-planes separation has been proposed in [87] as shown in Fig. 6(a). C-plane of a small cell is managed by a macro BS, and U-plane is served by the small cell itself that requires a new data path (S1-U) as a backhaul to connect to the core network. The macro BS is connected to the small cell via a new interface X3. The macrocell first sends an RRC message to a UE to measure channel conditions between the UE and the small cell for the connection establishment. After measuring channels, the UE then reports channel conditions to the macrocell. The macrocell then asks the small cell via backhaul links for its preparation to serve the UE. Once the small cell confirms with a positive response for its preparation to the macrocell, the macrocell then initiates to the UE an RRC connection set-up request from the small cell to the UE. The UE then requests for a connection to the small cell using the random access (RA) procedure with preamble. The small cell then responds to the UE's RA request, and the UE then sends the RRC connection set-up (between the small cell and the UE) confirmation message to the 
macrocell. User data are now sent directly via S1-U interface from the core network to the small cell, and the small cell then sends user data to the UE.

Decoupling downlink and uplink: In downlink of HetNets, different BSs have different transmission powers with a difference of more than $20 \mathrm{~dB}$ between a macro BS and a femto BS, and hence their coverage vary accordingly. However, in uplink, transmission powers from UEs are almost the same. In downlink, the maximum SINR region follows the transmission power of each BS, i.e., a macro BS with the largest, and a femto BS with the smallest. However, this is not the case in uplink where the maximum SINR region of a macro BS suffers the most, and the maximum SINR region of a femto BS can surpass the coverage of a macro BS [89]. This is because UEs are comparatively much closer to a small cell BS than a macro BS. Hence, a UE may have a good coverage for uplink from one BS and from another BS for downlink. Further, when UEs are served by different BSs for uplink transmissions, they may cause interferences to one another if UEs are assigned the same resources in uplink. Hence, classical interference models considering symmetry in uplink and downlink are not directly applicable for HetNets. So, uplink and downlink should be considered as separate networks along with new interference models for HetNets with asymmetry in transmission power and irregularity in deployment. Figure 6(b) shows an example scenario of this type of network architectures.

Decoupling baseband processing unit and node: In this decoupling approach [90], by introducing virtualization concepts on BSs, baseband signal processing hardware unit (BBU) is decoupled from its node, i.e., BS [8], and all BBUs are aggregated centrally into a virtual BS pool. Centralized BBU, cooperative radio with distributed antenna based RRHs and real-time cloud infrastructure radio access network (C-RAN) can address requirements of $5 \mathrm{G}$ networks. With a centralized BBU, a reduction in site costs and with RRHs, an increase in spectrum efficiency can be achieved. Further, with real-time cloud infrastructure and BS virtualization, dynamics in resource allocation, a reduction in power consumption, and an increase in infrastructure utilization can be achieved. C-RAN is an alternative approach to current networks and is targeting to the most typical HetNets scenario [90]. Based on a layer-wise (both control and data planes) functional splitting between a BBU and RRHs, full centralization and partial centralization of C-RAN can be realized. In full centralization of CRAN, baseband (i.e., layer 1), layer 2 and layer 3 are incorporated in a BBU. However, in partial virtualization of C-RAN, layer 1 functionalities are left with RRHs. In either case, a C-RAN consists of three main components [90], namely, a BBU which is incorporated with a high-performance programmable processor and a real-time virtualization technology, distributed RRHs which are integrated with antennas, and high speed low latency optical fibers that connect all RRHs to the BBU. Figure 6(c) shows an example C-RAN architecture [90].

\subsection{Logically Centralized Programmable Control Network Platform}

SDN is a new networking paradigm which provides flexibility, simplicity, and evolvability in network operation, control, and management with a software platform. It principles on separating network intelligences to a logically centralized entity called controller from network processing units called data plane switches to simplify policy enforcement and network configuration and evolution [91]. Though the SDN principle was initially evolved around wired networks, recently it has got a significant momentum in wireless networks. Numerous research projects on wireless SDN (WSDN) are ongoing, and research approaches are proposed to address several issues of WSDN.

\subsubsection{Conventional SDN concept}

The concept of SDN is based on split architecture with the following characteristics [1]

1) Network control functions are separated from network forwarding functions.

2) Network intelligence is moved to a logically centralized single entity called SDN controller.

3) Controller maintains global abstracted network views on which control and management applications work.

4) SDN controller communicates with forwarding nodes using a standard protocol, e.g., OpenFlow.

5) Network operators can control and manage network requirements through SDN control and the management programs in SDN controller to be free from vendor agnostic processes. 


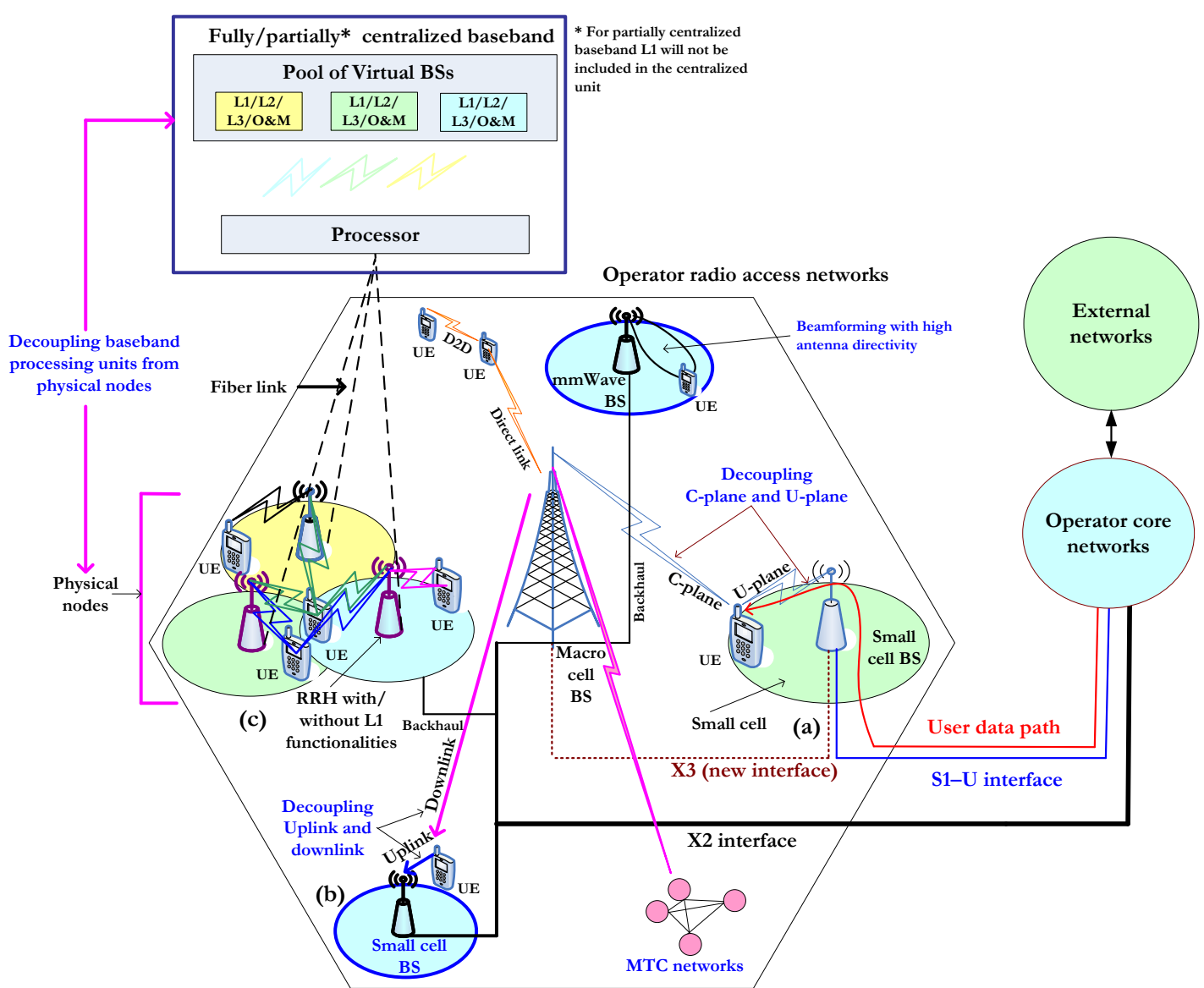

Fig. 6. A device-centric network architectural model for 5G networks.

Because of programmable features of SDN controller, these networks are referred to as software defined [92]. Figure 7 [93] shows a typical SDN architecture. SDN consists of three planes: a forwarding plane that includes forwarding elements, a control plane that includes network operating system and network hypervisor, and an application plane that includes network control and management applications. In addition, there are two interfaces: southbound (e.g., OpenFlow) and northbound (e.g., XML) interfaces. In the following, we give an overview on components of an SDN architecture.

A network operating system (NOS), like an ordinary computer operating system (OS), provides an ability to observe and control the network [94]. A NOS also keeps network states and provides a global view to the controller. The NOS and applications run on servers. POX, an NOS written in python, is an example of NOS. Applications are control and management programs that are usually implemented on top of the NOS and perform all control and management tasks. The global network view contains results of the NOS, and applications use it for control and management decisions. Example applications are routing and mobility management. A network hypervisor is used to virtualize physical resources into a number of virtual resources such that multiple users can use the same physical resources concurrently without intervening one another. A hypervisor is a software that is installed on a server. An example hypervisor is FlowVisor [95] that acts as a proxy such that all traffic to and from the controller and forwarding switches pass through the FlowVisor in order to enforce a proper policy on packets in each flow to provide an isolation between virtual network resources.

A southbound interface provides a necessary medium to communicate the controller with forwarding switches. A well-accepted southbound interface is OpenFlow. OpenFlow is a protocol that provides a way to communicate the controller with data paths using match-action rules [96]. When a packet arrives at a data path switch, its packet header is first matched with a flow entry in a flow table resided in each data path switch, and a corresponding action is taken on the packet following OpenFlow specifications for match-action rules such as 
forward, drop, modify, or send the packet to the controller. A northbound interface allows applications to communicate with the NOS. There is no standardized northbound interface yet. XML can be used as a northbound interface. SDN forwarding switches are responsible for switching a packet from an ingress port to an egress port. An example forwarding switch is OpenFlow switch that contains a forwarding table incorporating a number of flow entries. Each flow entry has three fields: a packet header defining a flow, an action defining how a packet should be processed, and statistics that keep record on the number of bytes and packets in each flow and the time since the last packet matched a flow [97]. Each flow is controlled by the controller.

\subsubsection{WSDN}

In wireless networking, there is no single dominant technology. Rather several technologies, e.g., cellular, WiFi, and worldwide interoperability for microwave access (WiMAX), are deployed in a particular scenario. Diversification in technology, distributed network management paradigm, unpredictable wireless medium, and multitude user requirements make current wireless networks hard to manage. SDN with its capability to separate control plane from data plane and to control data plane by providing a physical data plane network abstraction to control and management programs can provide flexibility and simplicity in wireless network control and management tasks. More importantly, it is the physical network abstraction which is the key in SDN that makes a significant impact on decisions of the controller and hence the overall efficiency. Before we discuss applications of SDN to wireless networks, we justify the following properties of SDN regarding wireless technologies.

Property 1 - Control plane and data plane can be separated: In current wireless distributed networking paradigms, network intelligences and processing functionalities of most network entities (e.g., SGW, PGW, and RAN in LTE) are distributed across the network. Through a proper interface (e.g., OpenFlow), the intelligence part of these network entities can be separated from the processing part, and be moved to a logically centralized entity - the controller. All management and decision applications can be implemented on top of the controller and can communicate with physical user-plane of these entities through a proper interface (e.g., XML).

Property 2 - SDN controller is technology independent. SDN controller simply takes decisions based on an abstraction given to it. So, SDN controller is technology independent. Though underlying network abstractions of LTE, WiMAX and WiFi are different, this technology distinction does not have any impact on the controller operation since the controller works simply on whatever an underlying abstraction it is provided with. Given simply an abstraction of underlying networks, SDN controller takes necessary decisions based on operator's goals provided through applications.

Property 3 - Abstracted network, can be segmented, and modular implementation can be introduced: In mobile networks, based on geographical regions, e.g., urban and suburban, we can segment the global view of an underlying network. And since each region has different characteristics replicated in the abstraction of that part of the network, SDN controller can set modular implementation such that there is a module for each region: urbanmodule, suburban-module, rural-module, and dense HetNet-module. Each module is responsible for that particular region and updates itself according to a change in the network abstraction of that region. This can simplify the controller decision and network management tasks since if there is any change needed for a particular region, only the corresponding module needs to be updated by leaving the rest of the network update unchanged.

A number of WSDN architectures for LTE cellular [98-100] and WiFi/WiMAX [101] have been proposed, mostly by separating access networks from core networks. Few LTE architectures leave legacy LTE networks almost unmodified while others propose changes on current networks and are explained in the following.

WSDN architectures without changing existing LTE core networks: A number of architectures have been proposed in [98] based on an integration of SDN with core network entities, e.g., MME, SGW, and PGW. SDN can be integrated as part of either MME for more awareness of mobility requirements, or it can be integrated as part of SGW and PGW to control transport networks. One of these proposed architectures is based on decoupling logical and data planes of SGW and PGW, as shown in Fig. 8 [98]. Logical parts of SGW and PGW are separated and are integrated with an SDN controller. The controller manages data planes of SGW and PGW. 
The MME interacts with logical parts of SGW and PGW. The rest of the elements in the network are kept unchanged.

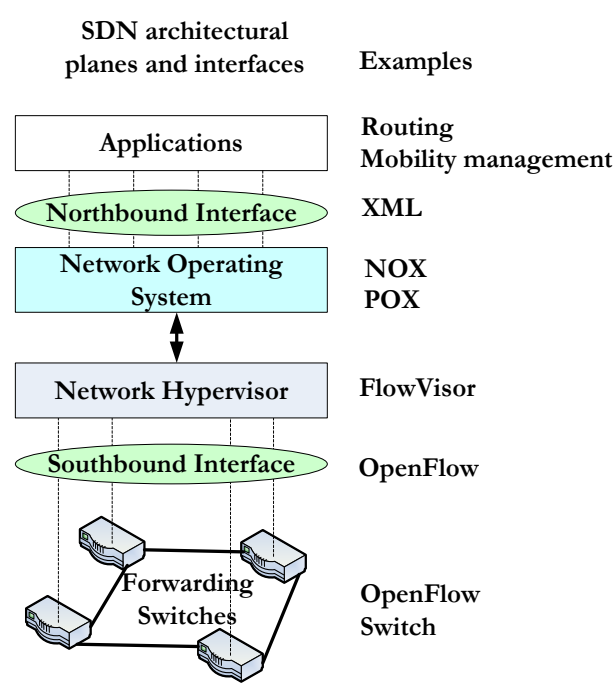

Fig. 7. Typical SDN architecture [93].

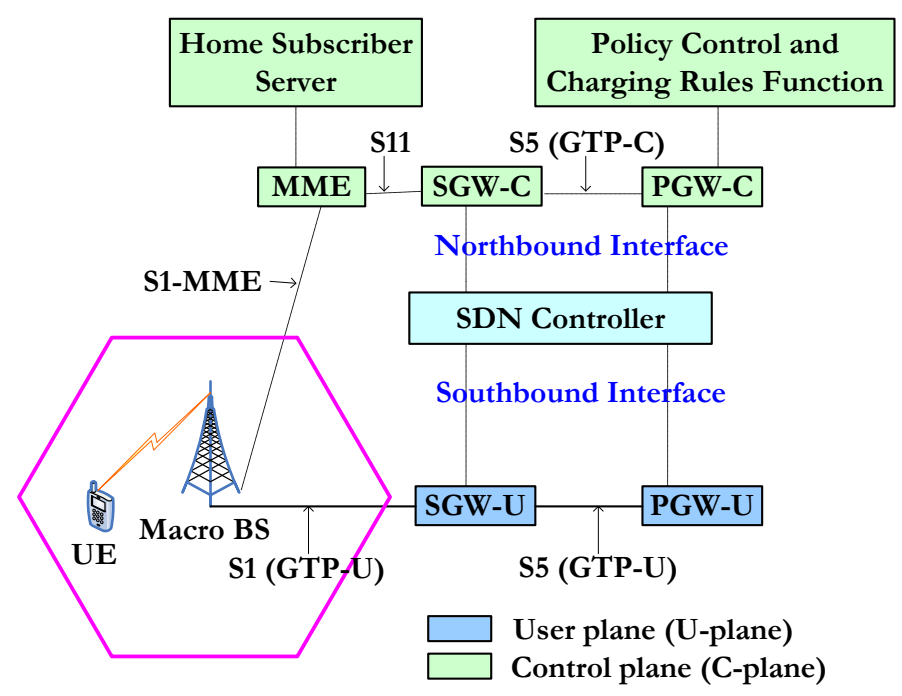

Fig. 8. Integration of SDN with S/P-GW [98].

WSDN architectures with changing existing LTE core networks: Softcell architecture has been proposed in [99] which considers changing current LTE core networks by removing core network elements such as SGW, PGW and point-to-point tunneling (Fig. 9). Instead, it considers supporting stateful middleboxes such that all packets in both directions of a connection must traverse the same instance. A controller implements high level service policy to direct traffic through middleboxes by using switch level rules. Each BS is associated with an access switch that is responsible for fine-grained packet classifications on a UE's traffic. The rest of the network consists of core switches, including a few gateway switches. Core switches are responsible for traffic forwarding functions through appropriate middleboxes [99]. The controller directs traffic over the network and middlebox paths based on service policies which are abstracted at a high level depending on subscriber's attributes and applications. A service policy includes multiple clauses that specify which traffic should be handled in what way. An example service policy clause is: VoIP traffic must go through an echo canceller followed by a firewall.

WSDN architectures on existing LTE access networks: SoftRAN architecture which is a result of an application of SDN principle on the LTE radio access network (RAN) is shown in Fig. 10 [100]. SoftRAN is a software defined centralized control plane for access networks that abstracts all physical BSs in a geographical area as a virtual big BS. Rather than controlling radio resources of each BS by itself in a distributed manner, all resources are allocated by a central controller among neighboring BSs. Radio resources are abstracted in three dimensions, including space (BS identifier), time, and frequency and are programmed by a logically centralized controller. In each time-frequency block, the controller needs to make a decision that is conveyed to each BS, and to assign an appropriate transmission power and the flow to be served by a BS. A centralized controller receives local network states periodically from all BSs in a local geographical area. The controller then updates and maintains the global network state in the form of a database called RAN Information Base (RIB) that contains information on interference maps (in the form of weighted graph), flow records (e.g., in the form of the number of bytes transmitted), and network operator's preferences (e.g., for priority services) which is accessed by various control modules to take decisions on the radio resource management. 


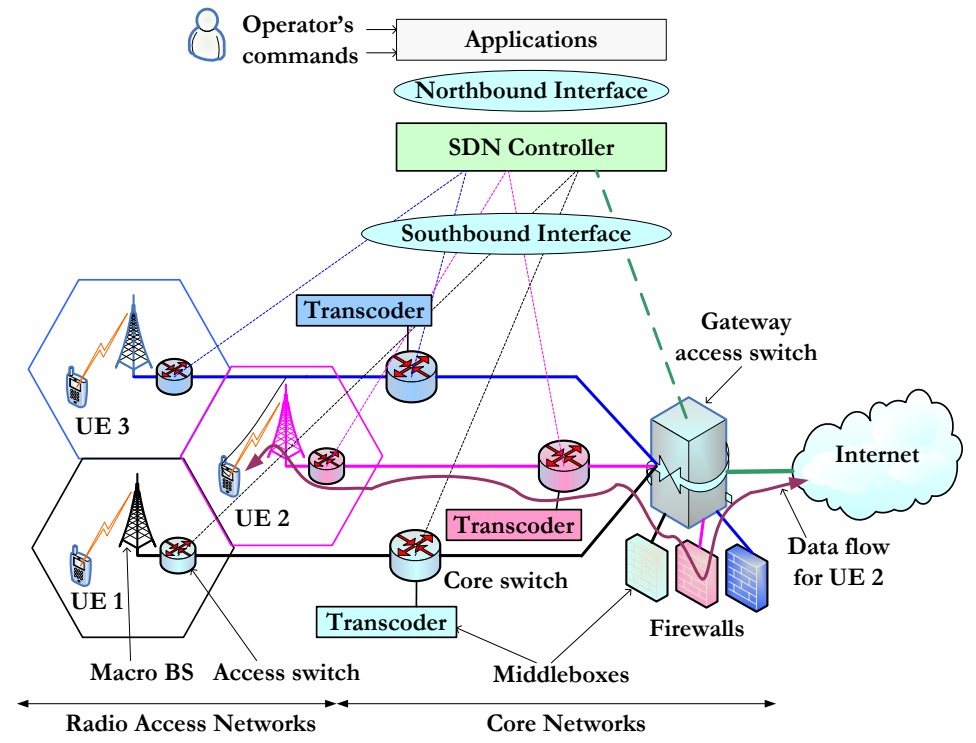

Fig. 9. Softcell network architecture [99].

WSDN Architectures on WiFi/WiMAX networks: OpenRoads or OpenFlow Wireless is a mobile wireless platform for experimental network researches and realistic deployment of networks and services using virtualization techniques as shown in Fig. 11 [101]. OpenFlow Wireless uses OpenFlow protocols to separate control plane from an underlying data path. A network hypervisor called FlowVisor is used to virtualize data plane to create network slices and to provide an isolation between slices such that multiple experiments can coexist and run in parallel with production networks without any intervention. In addition, a SNMPVisor is also used to configure radio specific problems [101]. All control and management applications communicate with the controller with a standard interface, and their decisions are conveyed to data paths by the OpenFlow protocol.

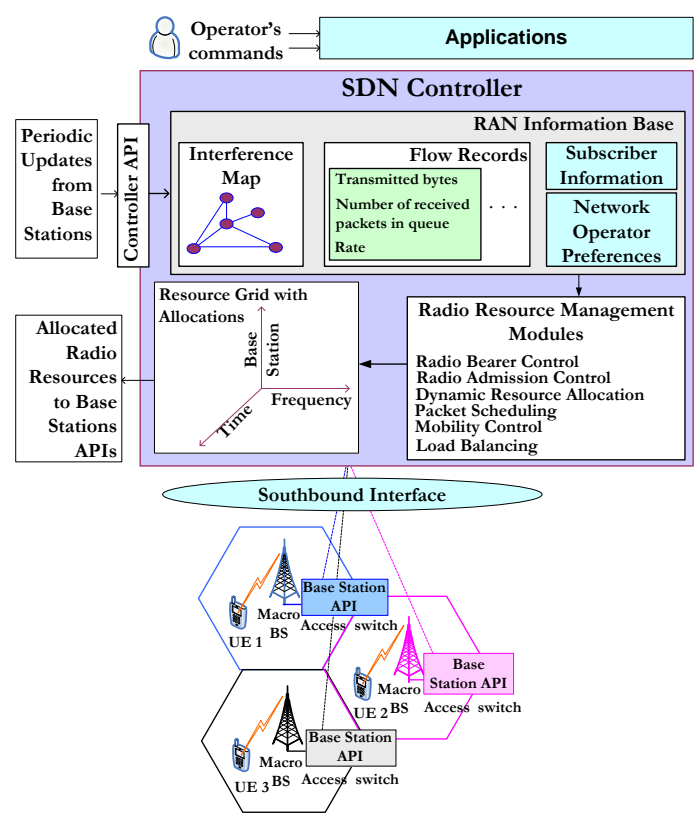

Fig. 10. SoftRAN architecture [100].

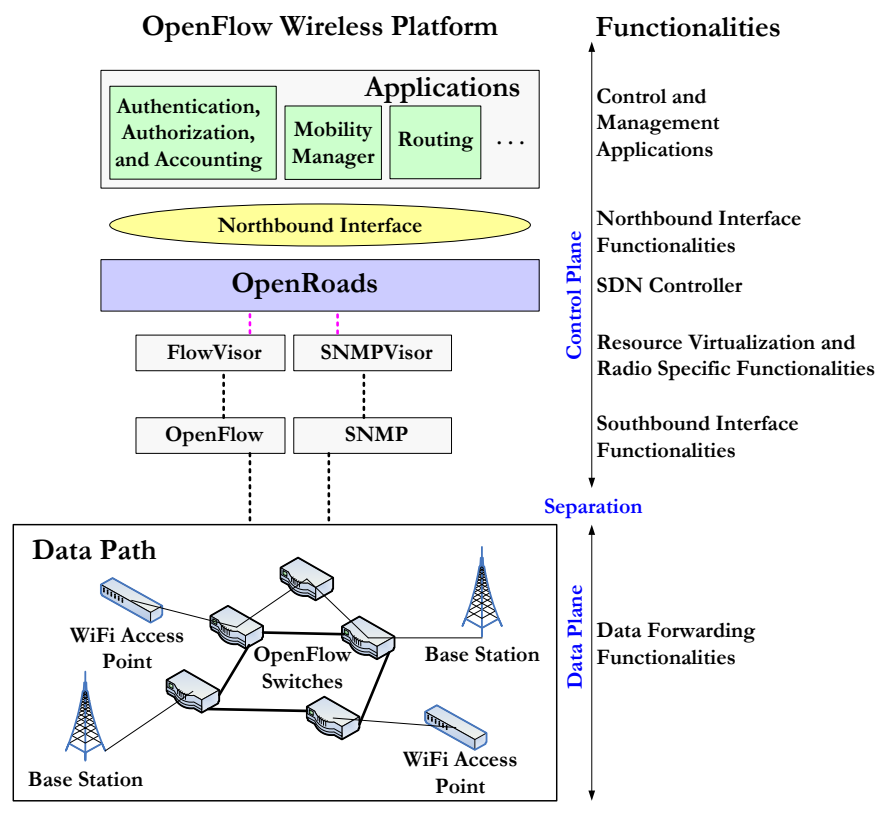

Fig. 11. OpenFlow Wireless architecture [101]. 


\subsubsection{WSDN implementation requirements and challenges}

Even though an adoption of SDN concept is highly desirable for wireless networks, there are several challenges that are to be addressed for the viability of WSDN from experimentation through technology development to policy level. A few of these requirements and challenges are outlined below.

1) $\mathrm{SDN}$ was developed with wired networks in mind. But characteristics of a wireless medium is far different and unpredictable. Adopting wireless medium characteristics in SDN is very challenging and requires considerable researches on this issue.

2) SDN is now in its early stage, and hence most elements of SDN are either under development or not broadly available in commercial markets. For example, the popular OpenFlow protocol specification is under the developing phase and still does not provide all radio configuration related functionalities.

3) $\mathrm{SDN}$ has so far been proposed to be integrated with current $4 \mathrm{G}$ mobile architectures without major changes on current networks (e.g., as proposed in [102]). Full advantages from the adoption of SDN is yet to be proven toward a higher $5 \mathrm{G}$ target and beyond.

4) Technology-specific bindings of virtualization are important due to a need to preserve efficiency in an unpredictable multi-user multi-access wireless medium [103]. It is also important to understand that not all these technologies benefit equally from various wireless virtualization perspectives [103]. Benefits of virtualization are mostly apparent in technologies where the bandwidth and the supported number of users are relatively high, leaving enough room for dynamic sharing of resources, such as in 802.11 WLAN and cellular networks.

5) OpenFlow does not provide any feature to configure data path elements, e.g., transmission power management and channel allocation. Therefore, an efficient wireless resource allocation and interference management cannot be achieved without more enhancements in the radio hardware. A comparative analysis for distributed and centralized network control programming approaches are given in Table 4.

Table 4. A comparative analysis for network control programming approaches.

\begin{tabular}{|c|c|c|c|}
\hline \multirow{2}{*}{ Features } & \multicolumn{2}{|c|}{$\begin{array}{l}\text { Logically distributed programmable } \\
\text { control networks }\end{array}$} & \multirow{2}{*}{$\begin{array}{c}\text { Logically centralized } \\
\text { programmable control } \\
\text { networks }\end{array}$} \\
\hline & $\begin{array}{l}\text { Cell-centric } \\
\text { control }\end{array}$ & $\begin{array}{l}\text { Device-centric } \\
\text { control }\end{array}$ & \\
\hline Network architecture & Cell-centric & Device-centric & WSDN controller-centric \\
\hline Network programming & $\begin{array}{l}\text { Networking device } \\
\text { level }\end{array}$ & $\begin{array}{l}\text { Networking device } \\
\text { level }\end{array}$ & WSDN controller level \\
\hline Network control assessment & $\begin{array}{l}\text { Based on a local } \\
\text { network view }\end{array}$ & $\begin{array}{l}\text { Based on a local } \\
\text { network view }\end{array}$ & $\begin{array}{l}\text { Based on a global } \\
\text { network view }\end{array}$ \\
\hline Network control mechanism & $\begin{array}{l}\text { Both logically and } \\
\text { physically distributed }\end{array}$ & $\begin{array}{l}\text { Both logically and } \\
\text { physically distributed }\end{array}$ & $\begin{array}{l}\text { Logically centralized but } \\
\text { physically distributed }\end{array}$ \\
\hline $\begin{array}{l}\text { Control plane and data plane } \\
\text { of a networking device }\end{array}$ & $\begin{array}{l}\text { Integrated in the same } \\
\text { networking device }\end{array}$ & $\begin{array}{l}\text { Integrated in the same } \\
\text { networking device }\end{array}$ & $\begin{array}{l}\text { Control plane is } \\
\text { separated from data } \\
\text { plane }\end{array}$ \\
\hline UE control mechanism & $\begin{array}{l}\text { Fully networked } \\
\text { controlled }\end{array}$ & $\begin{array}{l}\text { Both network and } \\
\text { device controlled }\end{array}$ & $\begin{array}{l}\text { Fully controlled by a } \\
\text { WSDN controller }\end{array}$ \\
\hline $\begin{array}{l}\text { Control and management of } \\
\text { network requirements }\end{array}$ & Vendor agnostic & Vendor agnostic & $\begin{array}{l}\text { Free from vendor } \\
\text { agnostic processes }\end{array}$ \\
\hline
\end{tabular}




\section{Backhaul Network Platform and Synchronization}

\subsection{Backhaul Networks, Deployment Scenarios and Backhaul Solutions}

5G networks will be denser than current HetNets in LTE-Advanced systems. In such a complex HetNet, a tight integration and a high level of cooperation between cells are crucial to address several significant and unavoidable issues such as interference management and load balancing. Backhaul networks will play a significant role on connecting BSs in a dense HetNet and providing a channel to communicate and to cooperate one BS to another. Backhaul networks take a considerable share of the total cost of ownership of the network. Hence, backhaul solutions should be cost-effective, easy to install, highly scalable and flexible, and not be a barrier to performances of HetNets [104]. However, reusing the same spectrum between spatially separated cells puts much requirements on delay, delay variation, and synchronization, particularly between a macrocell and a small cell.

There are a number of backhaul solutions such as microwave links using above $20 \mathrm{GHz}$ frequency bands [105], microwave links using sub-6 GHz frequency bands, point-to-point optical fiber, point-to-multipoint (PMP) optical fiber, category 5/6 LAN, and DSL. Backhaul solutions vary with requirements such as scalability, cost-effectiveness, and QoE. Scalability of a network can be achieved through a well-defined and carefully selected set of these backhaul technologies. By considering a common approach for traffic management by using single-vendor provided solutions, the total cost of ownership for the small cell layer can be made one-half from, e.g., reduced infrastructures by the proper coordination [106] to address the cost issue. If a backhaul solution needs to address QoE, one way to do so is to ensure a proper coordination between radio nodes and layers which require a high performance end-to-end backhaul solution such as optical fiber or LOS microwave link, particularly for links from macrocells whenever possible. If a fixed backhaul solution is not available, wireless links can be used as a default choice, and NLOS can also be considered for a greater degree of freedom.

For outdoor deployments, wireless and optical fiber backhauls are good choices and for indoor deployments, reuse of existing copper and optical fiber can be considered. Practical deployment scenarios, e.g., on city street, there are mainly two deployment scenarios, namely, an indoor environment (e.g., indoor hotspot) and an outdoor environment (e.g., bus stop). For an indoor hotspot, usually a picocell is deployed, and can be connected to a macrocell over an existing DSL line backhaul. However, based on capacity demands and environment profiles, optical fiber or LOS microwave can be considered. For almost all other indoor small cells, almost any backhaul can be used. For an in-building scenario, telephony cable and copper category $5 / 6$ cable are seem to be the most used backhauls. For an outdoor microcell, it should be connected to the macrocell via a LOS or a high performing NLOS backhaul.

\subsection{Advanced Technologies and Impact on Backhaul Networks}

Since dense small cells, massive MIMO, mmWave spectrum, and cooperative communications are considered as the major technologies for shaping the high data demand of $5 \mathrm{G}$ networks, each of these technologies imposes burden on backhaul networks. This is because a high data generated from using these technologies must be transferred to the core network. Deploying small cells densely will generate a large signaling overhead from frequent handover, high interference, and load unbalancing. A tighter coordination between small cells will generate a large overhead from information which is exchanged from one to another. When coordination is adopted for a joint CoMP, user data along with other overheads are to be exchanged via backhaul interfaces among cooperating nodes. Further, in cooperative MIMO systems, multiple base stations cooperate to exchange resource allocation and scheduling information decisions that result in more signaling overheads on backhauls. In massive MIMO systems, 100 or even greater number of antennas may be installed on the same site which requires feedback reports for pre-coding matrices, CSIs, and RIs from UEs. As the number of antennas in a massive MIMO system increases, so are feedback reports. Since mmWave communications are suitable for short distance communications, more links on mmWave frequencies are to be deployed to cover the same area, and hence more signaling overhead on backhauls will be required to communicate and to 
manage on these high frequencies. Typical backhaul transmission technologies under various aspects are listed in Table 5 where the symbol " $\mathrm{X}$ " denotes a typical use of the respective backhaul to an aspect.

Table 5. Typical backhaul transmission technologies.

\begin{tabular}{lcccc}
\multicolumn{1}{c}{ Aspects } & $\begin{array}{c}\text { WDM/ point-to- } \\
\text { point } \\
\text { optical fiber }\end{array}$ & $\begin{array}{c}\text { LOS/NLOS } \\
\text { microwave }\end{array}$ & LOS mmWave & $\begin{array}{c}\text { DSL/ } \\
\text { copper cable }\end{array}$ \\
\hline High capacity & $\mathrm{X}$ & $\mathrm{X}$ & $\mathrm{X}$ & \\
Low delay & $\mathrm{X}$ & $\mathrm{X}$ & $\mathrm{X}$ & $\mathrm{X}$ \\
Ease of installation & & $\mathrm{X}$ & $\mathrm{X}$ & $\mathrm{X}$ \\
Cost-effectiveness & $\mathrm{X}$ & $\mathrm{X}$ & $\mathrm{X}$ & \\
High QoE & $\mathrm{X}$ & $\mathrm{X}$ & $\mathrm{X}$ & $\mathrm{X}$ \\
Long distance link & $\mathrm{X}$ & $\mathrm{X}$ & $\mathrm{X}$ \\
Short distance link & $\mathrm{X}$ & $\mathrm{X}$ & $\mathrm{X}$ & \\
Indoor coverage & $\mathrm{X}$ & & & \\
Outdoor coverage & & &
\end{tabular}

\subsection{Synchronization in HetNets}

Synchronization in HetNets is very important because of an unplanned deployment of small cells. Time synchronizations mainly affect the cooperation mechanism between cells for the coordinated transmissions among multiple BSs as well as for the alignment of these signals at a receiver. In addition, handovers between cells are time sensitive, and an accuracy of 1.5 micro seconds for time synchronization is required in a time division based LTE system [107]. There are mainly three links that may be utilized for time synchronization, namely, backbone, satellite, and cellular links [107]. Since small cells are typically connected to the core network via IP links, time synchronization (TS) could be possible to achieve by using IEEE 1588 protocol, i.e., precision time protocol (PTP) that employs time-stamped packets between a server and its clients. However, in the context of small cells, PTP may face a lot of difficulties in terms of synchronization error and new investments from deploying PTP-enabled routers throughout the paths between clients and a server.

The use of satellite source such as global positioning system (GPS) can provide a highly accurate time information over satellite links in both frequency and time. GPS is commonly equipped with TDD based macrocells. However, it requires a good GPS-enabled receiver. Since small cells such as femtocells are expected to be deployed mainly to cover indoor environments, a satellite signal strength is not good enough in an indoor coverage with a resultant long time to synchronize or possibly no synchronization at all. The use of cellular networks for time synchronization can be performed by network listening to the synchronization signals from neighboring cells, which are possibly synchronized already, to perform timing adjustments.

Network listening synchronization for small cells is specified in the 3GPP [107]. Small cells can listen to the primary synchronization signal (PSS) or the secondary synchronization signal (SSS) or the common reference signal from neighboring BSs to perform time synchronization. This approach is cost-effective since it does not impose any extra infrastructure requirement. However, if the link between a macrocell and a small cell does not exist, then network listening cannot be done. In this case, small cell networks can perform time synchronization in a distributive manner. Every small cell at each iteration detects the synchronization signal and sets a new time based on its own time and the time from its neighboring small cells. Eventually, within a short time, all small cells converge to the same time value. Typical time synchronization links under various aspects are listed in Table 6 where the symbol " $\mathrm{X}$ " denotes a typical use of the respective synchronization link to an aspect.

Table 6. Typical time synchronization links.

\begin{tabular}{lccc}
\hline \multicolumn{1}{c}{ Aspects } & Backbone & Cellular link & Satellite link \\
\hline Indoor small cell & & $\mathrm{X}$ & $\mathrm{X}$ \\
Outdoor small cell & & $\mathrm{X}$ & $\mathrm{X}$ \\
Macrocell & $\mathrm{X}$ & $\mathrm{X}$ & $\mathrm{X}$ \\
\hline
\end{tabular}




\section{Future Evolutionary Directions and Challenges Toward 5G Networks}

Trends of all these technologies in three evolutionary directions reveal that there is no single dominant approach that can address the $5 \mathrm{G}$ network capacity most efficiently. However, these enabling technologies will certainly be the foundation toward the evolution of $5 \mathrm{G}$ networks. Referring to the proposed $5 \mathrm{G}$ network evolution framework, $5 \mathrm{G}$ networks can evolve in two extreme ways, namely, disruptive evolution and gradual evolution. In disruptive evolution, all core elements such as PGW, SGW, MME, and HSS of existing LTEAdvanced networks will be replaced by SDN based forwarding data plane switches such as OpenFlow switches. An SDN controller will then control centrally network BSs [98]. This approach can add flexibility to networking and support a gradual introduction of high network throughputs. Further, since MME and SDN controller are tightly integrated, an efficient hand over management can be performed.

On the other hand, in gradual evolution, the evolution toward $5 \mathrm{G}$ networks is based on existing cellular networks such as LTE-Advanced which allows network operators for a gradual migration toward 5G. The 3GPP standardization bodies have been consistently working toward meeting $5 \mathrm{G}$ features through different releases of LTE-Advanced. LTE release 12 is an initiative toward such a direction. Current LTE-Advanced systems can either be evolved based on a distributed programming approach or a new centralized programming approach as discussed in Section 3. In distributed programming approach, the main advantage is that there will be no need to change existing control mechanisms since existing control mechanisms are already based on a distributed programming approach. Since $5 \mathrm{G}$ networks are expected to incorporate new technologies such as MTC, D2D, and dense deployment of small cells, the LTE-Advanced system is more likely to evolve toward device-centric network architecture rather than existing cell-centric ones. A device-centric architecture can simplify network control mechanisms for new technologies by introducing decoupling features such as $\mathrm{C} / \mathrm{U}$ planes separation, up/down links separation, and BBU separation from physical nodes. By employing the CRAN infrastructure, features such as virtual BS computational resource and processing into a central pool, collaborative radio, and real-time cloud computing can be introduced. It can provide a new control plane that can enable user-centric BS reconfiguration and selection to make aware of situations and applications by introducing service oriented resource scheduling and management [108].

In a centralized programming approach, there is a need for changing current control mechanisms and introducing new techniques such as virtualization. Existing access and core network elements will need to be enabled with southbound protocols such as OpenFlow protocol to separate control plane from data plane. An SDN controller will need to be set-up which can be implemented physically in either distributed or centralized manner. In addition, existing access and core network elements will be virtualized using virtualization techniques to introduce network sharing mechanisms. However, this approach of programming the LTEAdvanced network provides flexibility, simplicity, and evolvability in network operation, control, and management. Further, a user-centric practice for service demand based BS control can be performed for 5G networks. A BS can be considered as a collection of services, rather than a collection of radio resources as is considered in traditional networking, and is called as open basestation [109]. Each BS can be contextualized on user characteristics and their diverse applications and service demands under its coverage by an SDN controller. This user-centric service demand based BS control will allow a BS to address the real-time guarantees of a diverse set of applications and services to users in $5 \mathrm{G}$ networks.

Irrespective of network programming approaches, network nodes will play a significant role on $5 \mathrm{G}$ networks. Low power nodes or small cells such as femtocells, picocells have already been a part of LTEAdvanced systems to boost system capacity. A dense deployment of these small cells within the coverage of macrocells will be one of the key enablers to meet the high capacity demand of $5 \mathrm{G}$ networks. These small cells will be deployed ultra-densely where the coverage of one cell may overlap the other. Advanced interference mitigation techniques such as time-domain eICIC will address co-tier interferences. Further, cooperative communications such as CoMP will help reduce inter-cell interferences and improve cell-edge UE throughputs. To boost the 5G capacity further, advanced multi-antenna techniques such as massive MIMO and networked MIMO will be expected to employ both at macro BSs and small cell BSs. In addition, to increase system bandwidth, mmWave spectrums will likely be used in small cells of $5 \mathrm{G}$ networks. Further, a large bandwidth 
based mmWave backhaul will play a significant role on enabling a seamless coordination between heterogeneous nodes. Through time synchronization either by network listening for small cells using existing cellular networks or by a distributive manner by considering the time from neighboring small cells, small cells can converge to a common time. With all these advanced enabling technologies employed on existing LTEAdvanced networks, a gradual evolution toward 5G networks will more likely be a viable solution to meet the capacity demand by 2020. Though these enabling technologies under each direction of architectural evolution of $5 \mathrm{G}$ networks have shown their immense potentials, many of them bring up new research challenges that must be overcome to demonstrate their practicability to the real $5 \mathrm{G}$ network implementation. Hence, an extensive level of studies is yet to be carried out to address these challenges. A number of such challenges are mentioned in the followings to point out possible further research directions.

\section{Radio access network node and performance enabler perspectives:}

1) The interference phenomenon and degree of densification of ultra-dense small cell networks are not well understood and remain open research problems.

2) Since massive MIMO systems rely on channel responses from UEs, acquiring accurate channel responses by reusing pilots to neighboring cells results in pilot contamination which is one of the major bottlenecks of massive MIMO systems that needs further studies. In addition, since massive MIMO systems are mainly proposed for TDD, considerable studies on the co-existence of TDD-and FDD-based systems are to be carried out.

3) The channel characteristics and atmospheric effects [110] on mmWave spectrums limit the coverage of $\mathrm{mmW}$ Wave communications largely in outdoor environments. Though the mmWave spectrums are primarily considered for indoor small cells, with ensuring a proper coordination mechanism, mmWave communications would be potential candidates for backhaul networks to replace the high cost optical fibers. Comprehensive understanding on merits from employing mmWave communications for backhaul networks requires additional studies not only from the technical but also from economic perspectives. In addition, the $\mathrm{C} / \mathrm{U}$ planes decoupling mechanisms for indoor mmWave deployed small cells needs considerable researches to be carried out.

Network control programming platform perspectives:

1) The full advantage of adopting SDN into wireless networks, particularly $5 \mathrm{G}$ mobile networks, is not well understood and hence needs substantial researches on this issue.

2) A common understanding and trade-off that considers multiple facets such as network control and management, access network performances, backhaul network overheads of distributed and centralized programmable networks is a worthwhile potential research direction.

3) Though several decoupling features of the new device-centric networks have been proposed in literatures, a significant level of practical demonstrations is essential to reinforce the viability of these features.

Backhaul network platform and synchronization perspectives:

1) The effect of ultra-densification of small cells on backhaul networks needs to be studied comprehensively under a number of constraints, e.g. environmental profile, UE density per unit area. Relative merits of one of the backhaul solutions to another both technically and economically need to be investigated.

2) Since the synchronization of small cells plays an important role on random placements and effective performances of small cells, the development of new synchronization techniques that is more contextualized for indoor ultra-dense small cell networks is a prospective research direction. 


\section{Conclusion}

An ever growing number of mobile users and usage of high data rate applications via cellular networks are expected to create a challenge on meeting the capacity demand of future $5 \mathrm{G}$ networks. Towards addressing the high capacity of $5 \mathrm{G}$ networks, a number of advanced enabling technologies have been proposed in literature. Until now, there is no clear direction on how the envisaged high capacity of $5 \mathrm{G}$ networks can be achieved by employing these enabling technologies. In this paper, we have addressed this issue by developing an evolution framework for $5 \mathrm{G}$ networks that consists of three evolutionary directions, specifically, radio access network node and performance enabler, network control programming platform, and backhaul network platform and synchronization. The framework has been developed in both an abstract level as well as a detailed one. We have carried out an extensive literature survey on enabling technologies in each evolutionary direction from a number of viewpoints. A rigorous comparative analysis of the major enabling technologies have been carried out under each evolutionary direction. Finally, future evolutionary directions and challenges toward 5G networks have been discussed in terms of a gradual evolution of existing LTE-Advanced networks and a disruptive evolution by changing existing networks. This survey will give an overall evolutionary trend in term of enabling technologies toward $5 \mathrm{G}$ networks from various perspectives and serve to give an insight of what enabling technologies in a number of evolutionary paths are more likely to drive and constitute the architecture of $5 \mathrm{G}$ networks under various scenarios to achieve the target $5 \mathrm{G}$ capacity.

\section{Acknowledgments}

This research is funded by (1) the Graduate School of Chulalongkorn University through The 100 th anniversary Chulalongkorn University Fund for Doctoral Scholarship, and (2) the Department of Electrical Engineering, Chulalongkorn University through the Electrical Engineering Chulalongkorn University $\mathrm{PhD}$ (EECU-PhD) Honors Program Scholarship at Chulalongkorn University, Bangkok, Thailand. The authors would like to thank the anonymous reviewers for their valuable comments to help improve the quality of the paper.

\section{References}

[1] S. Tomovic, M. Pejanovic-Djurisic, and I. Radusinovic, "SDN based mobile networks: concepts and benefits," Wireless Pers. Commun., vol. 78, no. 3, pp. 1629-1644, Oct. 2014.

[2] CISCO, "Visual networking index," CISCO, USA, White Paper, Feb. 2014.

[3] J. G. Andrews, S. Buzzi, W. Choi, S. V. Hanly, A. Lozano, A. C. K. Soong, and J. C. Zhang, "What will 5G be?," IEEE J. Sel. Areas Commun., vol. 32, no. 6, pp. 1065-1082, June 2014.

[4] A. Osseiran, F. Boccardi, V. Braun, K. Kusume, P. Marsch, M. Maternia, O. Queseth, M. Schellmann, H. Schotten, H. Taoka, H. Tullberg, M. A. Uusitalo, B. Timus, and M. Fallgren, "Scenarios for 5G mobile and wireless communications: the vision of the METIS project," IEEE Commun. Mag., vol. 52, no. 5, pp. 26-35, May 2014.

[5] E. Hossain, M. Rasti, H. Tabassum, and A. Abdelnasser, "Evolution toward 5G multi-tier cellular wireless networks: an interference management perspective," IEEE Wireless Commun., vol. 21, no. 3, pp. 118-127, June 2014.

[6] C. X. Wang, F. Haider, X. Gao, X. H. You, Y. Yang, D. Yuan, H. M. Aggoune, H. Haas, S. Fletcher, and E. Hepsaydir, "Cellular architecture and key technologies for 5G wireless communication networks," IEEE Commun. Mag., vol. 52, no. 2, pp. 122-130, Feb. 2014.

[7] D. L. Perez, I. Güvenç, G. D. L. Roche, M. Kountouris, T. Q. S. Quek, and J. Zhang, "Enhanced intercell interference coordination challenges in heterogeneous networks," IEEE Wireless Commun., vol. 18, no. 3, pp. 22-30, June 2011.

[8] F. Boccardi, R. W. Heath Jr., A. Lozano, T. L. Marzetta, and P. Popovski, "Five disruptive technology directions for 5G," IEEE Commun. Mag., vol. 52, no. 2, pp. 74-80, Feb. 2014.

[9] W.H. Chin, Z. Fan and R. Haines, "Emerging technologies and research challenges for 5G wireless networks," IEEE Wireless Commun., vol. 21, no. 2, pp. 106-112, Apr. 2014. 
[10] E. Hossain and M. Hasan, " $5 \mathrm{G}$ cellular: key enabling technologies and research challenges," IEEE Instrum. Meas. Mag., vol. 18, no. 3, pp. 11-21, June 2015.

[11] 3GPP, "3rd Generation Partnership Project; technical specification group radio access network; evolved universal terrestrial radio access (E-UTRA); FDD home eNode B (HeNB) radio frequency (RF) requirements analysis (release 12)," 3GPP, Valbonne-France, TR 36.921, ver. 12.0.0, 2014.

[12] A. Golaup, M. Mustapha, and L. B. Patanapongpibul, "Femtocell access control strategy in UMTS and LTE," IEEE Commun. Mag., vol. 47, no. 9, pp. 117-123, Sep. 2009.

[13] M. Iwamura, H. Takahashi, and S. Nagata, "Relay technology in LTE-Advanced," NTT DOCOMO Technical Journal, vol. 12, no. 2, pp. 29-36, Sep. 2010.

[14] 3GPP, "3rd Generation Partnership Project; technical specification group radio access network; evolved universal terrestrial radio access (E-UTRA); further advancements for E-UTRA physical layer aspects (release 9)," 3GPP, Valbonne, France, TR 36.814, ver. 9.0.0, 2010.

[15] C. X. Wang, X. Hong, X. Ge, X. Cheng, G. Zhang, and J. Thompson, "Cooperative MIMO channel models: a survey," IEEE Commun. Mag., vol. 48, no. 2, pp. 80-87, Feb. 2010.

[16] O. N. C. Yilmaz, Z. Li, K. Valkealahti, M. A. Uusitalo, M. Moisio, P. Lundén, and C. Wijting, "Smart mobility management for D2D communications in 5G networks," IEEE Wireless Communications and Networking Conference Workshops (WCNCW), Istanbul, 6-9 Apr. 2014, pp. 219-223.

[17] K. Doppler, M. Rinne, C. Wijting, C. B. Ribeiro, and K. Hugl, "Device-to-device communication as an underlay to LTE-Advanced networks," IEEE Commun. Mag., vol. 47, no. 12, pp. 42-49, Dec. 2009.

[18] 3GPP, "Study on LTE device to device proximity services," Qualcomm Inc., RP-122009, Dec. 2012.

[19] 3GPP, "Technical specification group services and system aspects; feasibility study for proximity services (ProSe) (release 12)," 3GPP, Valbonne-France, TR 22.803, ver.12.2.0, June 2013.

[20] A. Zakrzewska, S. Ruepp, and M. S. Berger, "Towards converged 5G mobile networks-challenges and current trends," in Proceedings of the 2014 ITU Kaleidoscope Academic Conference: Living in a Converged World Impossible without Standards?, St. Petersburg, 3-5 June 2014, pp. 39-45.

[21] V. Goncalves and P. Dobbelaere, "Business scenarios for machine-to-machine mobile applications," in Proc. 2010 Ninth International Conference on Mobile Business and 2010 Ninth Global Mobility Roundtable (ICMBGMR), Athens, Greece, 13-15 June 2010, pp. 394-401.

[22] M. J. Shih, Y. C. Pang, G. Y. Lin, H. Y. Wei, and R. Vannithamby, "Performance evaluation for energyharvesting machine-type communication in LTE-A system," in Proc. 79th IEEE Vehicular Technology Conference (VTC Spring), Seoul, South Korea, May 2014, pp. 1-5.

[23] C. L. I, S. Han, Y. Chen, and G. Li, "Trillions of nodes for 5G!?," in Proc. IEEE/CIC International Conference on Communications in China (ICCC), Shanghai, China, 13-15 Oct. 2014, pp. 246-250.

[24] T. Taleb and A. Kunz, "Machine type communications in 3GPP networks: potential, challenges, and solutions," IEEE Commun. Mag., vol. 50, no. 3, pp. 178-184, Mar. 2012.

[25] G. Wu, S. Talwar, K. Johnsson, N. Himayat, and K. D. Johnson, "M2M: from mobile to embedded internet," IEEE Commun. Mag., vol. 49, no. 4, pp. 36-43, Apr. 2011.

[26] 3GPP "Service requirements for machine-type communications (MTC), stage 1, release 12," 3GPP, Valbonne-France, TS 22.368, ver. 12.3.0, 2013.

[27] D. Astely, E. Dahlman, G. Fodor, S. Parkvall, and J. Sachs, "LTE release 12 and beyond," IEEE Commun. Mag., vol. 51, no. 7, pp. 154-60, July 2013.

[28] ETSI, "Technical specification: machine-to-machine communications (M2M); functional architecture," ETSI, 650 Route des Lucioles, F-06921 Sophia Antipolis Cedex, France, TS 102 690, ver. 2.1.1, Oct. 2013.

[29] ETSI, "Architecture enhancements to facilitate communications with packet data networks and applications," ETSI, 650 Route des Lucioles, Sophia Antipolis Cedex, France, TS 123 682, ver. 11.4.0, 2013.

[30] V. Osa, C. Herranz, J. Monserrat, and X. Gelabert, "Implementing opportunistic spectrum access in LTE-Advanced," EURASIP J. Wireless Commun. Netw., vol. 2012, no. 3, pp. 1-17, Mar. 2012.

[31] Y. Chen and W. Wang, "Machine-to-machine communication in LTE-A," in Proc. 2010 IEEE $72^{\text {nd }}$ Vehicular Technology Conference Fall (VTC 2010-Fall), Ottawa, ON, 6-9 Sept. 2010, pp. 1-4. 
[32] M. Jo, T. Maksymyuk, R. L. Batista, T. F. Maciel, A. L. F. D. Almeida, and M. Klymash, "A survey of converging solutions for heterogeneous mobile networks," IEEE Wireless Commun., vol. 21, no. 6, pp. 54-62, Dec. 2014.

[33] A. Ghosh, R. Ratasuk, B. Mondal, N. Mangalvedhe, and T. Thomas, "LTE-Advanced: Next-generation wireless broadband technology," IEEE Wireless Commun., vol. 17, no. 3, pp. 10-22, June 2010.

[34] M. Hasan, E. Hossain and D. Niyato, "Random access for machine-to-machine communication in LTEAdvanced networks: issues and approaches," IEEE Commun. Mag., vol. 51, no. 6, pp. 86-93, June 2013.

[35] A. Elmangoush, H. Coskun, S. Wahle, and T. Magedanz, "Design aspects for a reference M2M communication platform for smart cities," in Proc. International Conference on Innovations in Information Technology (IIT), Abu Dhabi, Mar. 2013, pp. 204-209.

[36] R. Valli and P. Dananjayan, "Transmission power control in virtual MIMO wireless sensor network using game theoretic approach," in Proc. First International Conference on Computer Science and Information Technology, CCSIT Proceedings, Part II, Bangalore, India, Jan. 2011, pp. 535-545.

[37] S. Mumtaz, K. M. S. Huq, and J. Rodriguez, "Coordinated paradigm for D2D communications," 2014 IEEE INFOCOM Workshop on Green Cognitive Communications and Computing Networks, Toronto, ON, 27 Apr.-2 May 2014, pp. 718-723.

[38] N. Bhushan, J. Li, D. Malladi, R. Gilmore, D. Brenner, A. Damnjanovic, R. T. Sukhavasi, C. Patel, and S. Geirhofer, "Network densification: the dominant theme for wireless evolution into 5G," IEEE Commun. Mag., vol. 52, no. 2, pp. 82-89, Feb. 2014.

[39] V. Jungnickel, K. Manolakis, W. Zirwas, B. Panzner, V. Braun, M. Lossow, M. Sternad, R. Apelfröjd, and T. Svensson, "The role of small cells, coordinated multipoint, and massive MIMO in 5G," IEEE Commun. Mag., vol. 52, no. 5, pp. 44-51, May 2014.

[40] S. F. Yunas, M. Valkama, and J. Niemelä, "Spectral and energy efficiency of ultra-dense networks under different deployment strategies," IEEE Commun. Mag., vol. 53, no. 1, pp. 90-100, Jan. 2015.

[41] B. Soret, K. I. Pedersen, N. T. K. Jørgensen, and V. Fernández-López, "Interference coordination for dense wireless networks," IEEE Commun. Mag., vol. 53, no. 1, pp. 102-109, Jan. 2015.

[42] J. Xu, J. Wang, Y. Zhu, Y. Yang, X. Zheng, S. Wang, L. Liu, K. Horneman, and Y. Teng, "Cooperative distributed optimization for the hyper-dense small cell deployment," IEEE Commun. Mag., vol. 52, no. 5, pp. 61-67, May 2014.

[43] R. Q. Hu and Y. Qian, "An energy efficient and spectrum efficient wireless heterogeneous network framework for 5G systems," IEEE Commun. Mag., vol. 52, no. 5, pp. 94-101, May 2014.

[44] F. Letourneux, Y. Corre, E. Suteau, and Y. Lostanlen, "3D coverage analysis of LTE urban heterogeneous networks with dense femtocell deployments," EURASIP J. Wirel. Commun. Netw., Springer International Publishing AG, vol. 2012, no. 1, pp. 1-14, Oct. 2012.

[45] M. Condoluci, M. Dohler, G. Araniti, A. Molinaro, and K. Zheng, "Toward 5G dense HetNets: architectural advances for effective machine-type communications over femtocells," IEEE Commun. Mag., vol. 53, no. 1, pp. 134-141, Jan. 2015.

[46] W. Zhuang and M. Ismail, "Cooperation in wireless communication networks," IEEE Wireless Commun., vol. 19, no. 2, pp. 10-20, Apr. 2012.

[47] E. C. V. D. Meulen, “Three-terminal communication channels," Advanced Applied Probability, vol. 3, no. 1, pp. 120-154, 1971.

[48] T. M. Cover and A. E. Gamal, "Capacity theorems for the relay channel," IEEE Trans. Info. Theory, vol. 25, no. 5, pp. 572-584, Sept. 1979.

[49] Q. Li, R. Q. Hu, Y. Qian, and G. Wu, "Cooperative communications for wireless networks: techniques and applications in LTE-Advanced systems," IEEE Wireless Commun., vol. 19, no. 2, pp. 22-29, Apr. 2012.

[50] M. Sawahashi, Y. Kishiyama, A. Morimoto, D. Nishikawa, and M. Tanno, "Coordinated multipoint transmission/reception techniques for LTE-Advanced," IEEE Wireless Commun., vol. 17, no. 3, pp. 2634, June 2010.

[51] 3GPP, "Technical report 3rd Generation Partnership Project; technical specification group radio access network; coordinated multi-point operation for LTE physical layer aspects (release 11)," 3GPP, Valbonne-France, TR 36.819, ver. 11.2.0, Sep. 2013. 
[52] S. Sun, Q. Gao, Y. Peng, Y. Wang, and L. Song, "Interference management through CoMP in 3GPP LTE-Advanced networks," IEEE Wireless Commun., vol. 20, no. 1, pp. 59-66, Feb. 2013.

[53] NTT DOCOMO, "Performance of eICIC with control channel coverage limitation," 3GPP Std., Montreal, Canada, R1-103264, May 2010.

[54] D. Castanheira and A. Gameiro, "Distributed antenna system capacity scaling," IEEE Wireless Commun., vol. 17, no. 3, pp. 68-75, June 2010.

[55] X. Zhang, K. Sundaresan, M. A. Khojastepour, S. Rangarajan, and K. G. Shin, "NEMOx: Scalable network MIMO for wireless networks," in Proceedings of the 19th Annual International Conference on Mobile Computing \& Networking, MobiCom'13, Miami, FL, USA, Sep. 30 -Oct. 4, 2013, pp. 453-464.

[56] Z. Zong, H. Feng, T. Yang, and B. Hu, "Distributed framework of downlink CoMP MU-MIMO transmission with adaptive mode switch and power allocation," in Proc. 2014 International Conference on Computing, Networking and Communications, Wireless Communications Symposium, Honolulu, HI, Feb. 2014, pp. 611-615.

[57] E. G. Larsson, O. Edfors, F. Tufvesson, and T. L. Marzetta, "Massive MIMO for next generation wireless systems," IEEE Commun. Mag., vol. 52, no. 2, pp. 186-195, Feb. 2014.

[58] T. L. Marzetta, "Massive MIMO: An introduction," Bell Labs Tech. J., vol. 20, pp. 11-22, Mar. 2015.

[59] D. Qiao, Y. Wu, and Y. Chen, "Massive MIMO architecture for 5G networks: Co-located, or distributed?," in Proc. 2014 11th International Symposium on Wireless Communications Systems (ISWCS), Barcelona, 26-29 Aug. 2014, pp. 192-197.

[60] T.L. Marzetta, "Noncooperative cellular wireless with unlimited numbers of base station antennas," IEEE Trans. Wireless Commun., vol. 9, no. 11, pp. 3590-3600, Nov. 2010.

[61] T. S. Rappaport, S. Sun, R. Mayzus, H. Zhao, Y. Azar, K. Wang, G. N. Wong, J. K. Schulz, M. Samimi, and F. Gutierrez, "Millimeter wave mobile communications for 5G cellular: it will work!," IEEE Access, vol. 1, pp. 335-349, May 2013.

[62] T. S. Rappaport, "Introduction to wireless communication systems," Wireless Communications: Principles and Practice, $2^{\text {nd }}$ ed. Englewood Cliffs, NJ, USA: Prentice-Hall, 2002, ch. 1, pp. 1-24.

[63] F. Gutierrez, S. Agarwal, K. Parrish, and T. S. Rappaport, "On-chip integrated antenna structures in CMOS for 60 GHz WPAN systems," IEEE J. Sel. Areas Commun., vol. 27, no. 8, pp. 1367-1378, Oct. 2009.

[64] T. S. Rappaport, E. Ben-Dor, J. N. Murdock, and Y. Qiao, "38 GHz and $60 \mathrm{GHz}$ angle-dependent propagation for cellular and peer-to-peer wireless communications," in Proc. 2012 IEEE International Conference on Communications (ICC), Ottawa, ON, 10-15 June 2012, pp. 4568-4573.

[65] T. S. Rappaport, J. N. Murdock, and F. Gutierrez, "State of the art in $60 \mathrm{GHz}$ integrated circuits \& systems for wireless communications," in Proc. IEEE, Aug. 2011, vol. 99, no. 8, pp. 1390-1436.

[66] F. Rusek, D. Persson, B. K. Lau, E. G. Larsson, T. L. Marzetta, O. Edfors, and F. Tufvesson, "Scaling up MIMO: opportunities and challenges with very large arrays," IEEE Signal Process. Mag., vol. 30, no. 1, pp. 40-60, Jan. 2013.

[67] J. Qiao, X. Shen, J. W. Mark, Q. Shen, Y. He, and L. Lei, "Enabling device-to-device communications in millimeter-wave 5G cellular networks," IEEE Commun. Mag., vol. 53, no. 1, pp. 209-215, Jan. 2015.

[68] S. Geng, J. Kivinen, X. Zhao, and P. Vainikainen, "Millimeter-wave propagation channel characterization for short-range wireless communications," IEEE Trans. Vehic. Tech., vol. 58, no. 1, pp. 3-13, Jan. 2009.

[69] K. Zheng, F. Liu, L. Lei, C. Lin, and Y. Jiang, "Stochastic performance analysis of a wireless finite-state Markov channel," IEEE Trans. Wireless Commun., vol. 12, no. 2, pp. 782-93, Feb. 2013.

[70] Z. Pi and F. Khan, "An introduction to millimeter-wave mobile broadband systems," IEEE Commun. Mag., vol. 49, no. 6, pp. 101-107, June 2011.

[71] FCC, "Millimeter wave propagation: spectrum management implications," Office of Eng. and Tech., Bulletin no. 70, July 1997.

[72] S. Y. Seidel and H. W. Arnold, "Propagation measurements at $28 \mathrm{GHz}$ to investigate the performance of local multipoint distribution service (LMDS)," IEEE 1995 Global Telecommunications Conference (GLOBECOM'95), vol. 1, 14-16 Nov. 1995, pp. 754-757. 
[73] Q. Zhao and J. Li, "Rain attenuation in millimeter wave ranges," in Proc. $7^{\text {th }}$ IEEE International Symposium on Antennas, Propagation \& EM Theory, Guilin, 26-29 Oct. 2006, pp. 1-4.

[74] W. Roh, J. Y. Seol, J. Park, B. Lee, J. Lee, Y. Kim, J. Cho, K. Cheun, and F. Aryanfar, "Millimeter-wave beamforming as an enabling technology for $5 \mathrm{G}$ cellular communications: theoretical feasibility and prototype results," IEEE Commun. Mag., vol. 52, no. 2, pp. 106-113, Feb. 2014.

[75] V. Benedetto, M. Zaffaroni, D. Rui, and Q. Zhichao, "Huawei E-band RTN380-field trial report," Huawei Technologies Co., Ltd., China, Huawei White Paper, Oct. 2013.

[76] C. Dehos, J. L. González, A. D. Domenico, D. Kténas, and L. Dussopt, "Millimeter-wave access and backhauling: the solution to the exponential data traffic increase in $5 \mathrm{G}$ mobile communications systems?," IEEE Commun. Mag., vol. 52, no. 9, pp. 88-95, Sep. 2014.

[77] Q. Li, H. Niu, G. Wu, and R. Q. Hu, "Anchor-booster based heterogeneous networks with mmWave capable booster cells," in Proceedings of IEEE GLOBECOM Workshops, Atlanta, GA, Dec. 2013, pp. 93 98.

[78] R. Taori and A. Sridharan, "Point-to-multipoint in-band mmWave backhaul for 5G networks," IEEE Commun. Mag., vol. 53, no. 1, pp. 195-201, Jan. 2015.

[79] M. Paolini, "Crucial economics for mobile data backhaul-an analysis of the total cost of ownership of point-to-point, point-to-multipoint, and fiber options," Senza Fili Consulting, White Paper, 2011.

[80] R. Webb, "Macrocell mobile backhaul equipment and services market share and forecasts," Infonetics Research, 2012.

[81] Innovation Observatory, "Spectrum and technology issues for microwave backhaul in Europe," Innovation Observatory Ltd, UK, White Paper, 2010.

[82] R. Schwartz and M. Rice, "Rethinking small cell backhaul: a business case analysis of cost-effective small cell backhaul network solutions," Wireless 20/20, White Paper, July 2012.

[83] E. Monturus. (2011). A Practical Look at LTE Backhaul Capacity Requirements. [Online]. Available: http://www. slideshare.net /allabout4g/apracticallookatltebackhaulrequirementspdf

[84] P. J. Pietraski, "The bandwidth crunch: can wireless technology meet the skyrocketing demand for mobile data," IEEE Long Island Systems, Applications and Technology Conference (LISAT), Farmingdale, NY, 6 May 2011, pp. 1-6.

[85] D. Kreutz, F. M. V. Ramos, P. E. Ver1'ssimo, C. E. Rothenberg, S. Azodolmolky, and S. Uhlig, "Software-defined networking: a comprehensive survey," in Proc. IEEE, Jan. 2015, vol. 103, no. 1, pp. 14-76.

[86] T. Koponen, M. Casado, N. Gude, J. Stribling, L. Poutievski, M. Zhu, R. Ramanathan, Y. Iwata, H. Inoue, T. Hama, and S. Shenker, "Onix: A distributed control platform for large-scale production networks," in Proceedings of the 9th USENIX Conference on Operating Systems Design and Implementation, Ser. OSDI'10, Berkeley, CA, USA: USENIX Association, 2010, pp. 1-6.

[87] H. Ishii, Y. Kishiyama, and H. Takahashi, "A novel architecture for LTE-B: C-Plane/U-Plane split and phantom cell concept," in Proceedings of IEEE GLOBECOM Workshop, Anaheim, CA, Dec. 2012, pp. 624-630.

[88] 3GPP, "Evolved universal terrestrial radio access (E-UTRA) and evolved universal terrestrial radio access network (EUTRAN); overall description," 3GPP, Valbonne-France, TS 36.300, ver. 10.7.0, Mar. 2012.

[89] J. Andrews, “The seven ways HetNets are a paradigm shift," IEEE Commun. Mag., vol. 51, no. 3, pp. 136-144, Mar. 2013.

[90] China Mobile, "C-RAN: the road towards green RAN," China Mobile Research Institute, Beijing, China, White Paper, ver. 2.6, Sep. 2013.

[91] H. Kim and N. Feamster, "Improving network management with software defined networking," IEEE Commun. Mag., vol. 51, no. 2, pp. 114-119, Feb. 2013.

[92] J. Qadir, N. Ahmed, and N. Ahad. (2014). Building Programmable Wireless Networks: An Architectural Survey [Online]. Available: http://arxiv.org/pdf/1310.0251.pdf

[93] ON.LAB, Stanford University. (2014). ONS-SDN Controller [Online]. Available: http://onlab.us/tools.html 
[94] N. Gude, T. Koponen, J. Pettit, B. Pfaff, M. Casado, N. McKeown, and S. Shenker, "NOX: Towards an operating system for networks," ACM SIGCOMM Computer Commun. Review, vol. 38, no. 3, pp. 105-110, July 2008.

[95] R. Sherwood, G. Gibb, K. K. Yap, G. Appenzeller, M. Casado, N. McKeown, and G. Parulkar. (2009). FlowVisor: A Network Virtualization Layer [Online]. Available: http://OpenFlowSwitch.org/downloads/technicalreports/openflow-tr-2009-1-flowvisor.pdf

[96] K. K. Yap, R. Sherwood, M. Kobayashi, T. Y. Huang, M. Chan, N. Handigol, N. McKeown, and G. Parulkar, "Blueprint for introducing innovation into wireless mobile networks," in VISA 2010, Proceedings of the Second ACM SIGCOMM Workshop on Virtualized Infrastructure Systems and Architectures, NY, USA, Sep. 3, 2010, pp. 25-32.

[97] N. McKeown, T. Anderson, H. Balakrishnan, G. Parulkar, L. Peterson, J. Rexford, S. Shenker, and J. Turner, "OpenFlow: Enabling innovation in campus networks," ACM SIGCOMM Computer Commun. Review, vol. 38, no. 2, pp. 69-74, Apr. 2008.

[98] J. Costa-Requena, "SDN integration in LTE mobile backhaul networks," in Proc. 2014 International Conference on Information Networking (ICOIN), Phuket, Feb. 2014, pp. 264-269.

[99] X. Jin, L. E. Li, L. Vanbever, and J. Rexford, "SoftCell: Scalable and flexible cellular core network architecture," Proceedings of the Ninth ACM Conference on Emerging Networking Experiments and Tecbnologies, CoNEXT'13, ACM, USA, 9-12 Dec. 2013, pp. 163-174.

[100] A. Gudipati, D. Perry, L. E. Li, and S. Katti, "SoftRAN: Software defined radio access network," in Proceedings of the Second ACM SIGCOMM Workshop on Hot Topics in Software Defined Networking, HotSDN'13, ACM, Hong Kong, China, Aug. 2013, pp. 25-30.

[101] K. K. Yap, M. Kobayashi, D. Underhill, S. Seetharaman, P. Kazemian, and N. McKeown, “The Stanford OpenRoads deployment," in Proceedings of the 4th ACM International Workshop on Experimental Evaluation and Characterization, WiNTECH'09, Beijing, China, Sep. 2009, pp. 59-66.

[102] M. R. Sama, S. B. H. Said, K. Guillouard, and L. Suciu, "Enabling network programmability in LTE/EPC architecture using OpenFlow," in Proc. 2014 12th International Symposium on Modelling and Optimization in Mobil, Ad Hoc, and Wireless Networks (WiOpt), IEEE, Hammamet, May 2014, pp. 389-396.

[103] H. Wen, "Virtualization and software-defined infrastructure framework for wireless access networks," M. S. thesis, ECE Dept., McGill University, Montreal, Canada, Apr. 2014.

[104] Ericsson, "It all comes back to backhaul", Ericsson, White Paper, Aug. 2014.

[105] Ericsson, "Non-line-of-sight microwave backhaul for small cells," Ericsson, Ericsson Review, Feb. 2013.

[106] Ericsson, "Heterogeneous networks," Ericsson, White Paper, Sep. 2014.

[107] J. Xu, J. Wang, Y. Zhu, Y. Yang, X. Zheng, S. Wang, L. Liu, K. Horneman, and Y. Teng, "Cooperative distributed optimization for the hyper-dense small cell deployment," IEEE Commun. Mag., vol. 52, no. 5, pp. 61-67, May 2014.

[108] J. Wu, Z. Zhang, Y. Hong, and Y. Wen, "Cloud radio access network (C-RAN): A primer," IEEE Network, vol. 29, no. 1, pp. 35-41, Jan./Feb. 2015.

[109] A. Gatherer (2015). Towards the open basestation (IEEE ComSoc CTN, Issue: Feb. 2015) [online]. Available: http://www.comsoc.org/ctn/ieee-comsoc-ctn-special-issue-towards-open-basestation

[110] S. M. Rajendrakumar and M. Karruppaswamy, "Analysis of link availability in FSO-OFDM system under various climatic conditions," Engineering Journal, vol. 19, no. 1, pp. 85-95, Jan. 2015. 